\title{
PARABOLIC CURVES OF DIFFEOMORPHISMS ASYMPTOTIC TO FORMAL INVARIANT CURVES
}

\author{
L. LÓPEZ-HERNANZ AND F. SANZ SÁNCHEZ
}

\begin{abstract}
We prove that if $F$ is a tangent to the identity diffeomorphism at $0 \in \mathbb{C}^{2}$ and $\Gamma$ is a formal invariant curve of $F$ then there exists a parabolic curve (attracting or repelling) of $F$ asymptotic to $\Gamma$. The result is a consequence of a more general one in arbitrary dimension, where we prove the existence of parabolic curves of a tangent to the identity diffeomorphism $F$ at $0 \in \mathbb{C}^{n}$ asymptotic to a given formal invariant curve under some additional conditions, expressed in terms of a reduction of $F$ to a special normal form by means of blow-ups and ramifications along the formal curve.
\end{abstract}

\section{INTRODUCTION}

In this paper we prove the following theorem.

Theorem 1. Let $F$ be a tangent to the identity diffeomorphism in $\left(\mathbb{C}^{2}, 0\right)$. Given a formal invariant curve $\Gamma$ of $F$ not contained in the set of fixed points, there exists at least one (attracting or repelling) parabolic curve for $F$ asymptotic to $\Gamma$.

So far, this result was only known when $\Gamma$ is of "Briot-Bouquet type" as we explain below.

A formal curve $\Gamma$ at $0 \in \mathbb{C}^{2}$ is a prime ideal of $\mathbb{C}[[x, y]]$ generated by a single irreducible formal series. It is invariant for $F$ if $g \circ F \in \Gamma$ for any $g \in \Gamma$. An attracting parabolic curve for $F$ is an injective holomorphic map $\varphi: \Delta \rightarrow \mathbb{C}^{2}$, where $\Delta \subset \mathbb{C}$ is a simply connected domain with $0 \in \partial \Delta$, which is continuous at 0 with $\varphi(0)=0$ and such that $\varphi(\Delta)$ is invariant and attracted to the origin under the iteration of $F$. A repelling parabolic curve for $F$ is an attracting parabolic curve for $F^{-1}$. We say that a parabolic curve $\varphi$ is asymptotic to $\Gamma$ if there is an irreducible formal parametrization $\gamma(s) \in \mathbb{C}[[s]]^{2}$ of $\Gamma$ such that $\varphi$ has $\gamma(s)$ as asymptotic expansion in $\Delta$ at $s=0$. In particular, $\varphi$ can be blown-up following the infinitely near points of $\Gamma$.

When $\Gamma$ is convergent, up to reduction of singularities of $\Gamma$, the existence of parabolic curves asymptotic to $\Gamma$ can be reduced to the case of dimension 1 , where it is always guaranteed by Leau and Fatou Flower Theorem (see [18, 15]).

The existence of parabolic curves asymptotic to $\Gamma$ when $\Gamma$ is of "Briot-Bouquet type" (its tangent line corresponds to a non-zero eigenvalue after reduction of singularities of the infinitesimal generator of $F$ ) follows from Écalle [14] and Hakim [17. (see also Abate et al. [1, 2] and Brochero et al. [6]). In fact, the first author has proved in [19] that these parabolic curves are the sums (in the sense of Ramis

First author partially supported by CNPq, Brazil, process 479134/2013-8. Both authors partially supported by Ministerio de Educación y Cultura, Spain, process MTM2010-15471, and by Programa Hispano-Brasileño de Cooperación Interuniversitaria, process PHB2010-0122-PC. 
summability theory) of $\Gamma$, so they can be recovered from $\Gamma$ by means of the BorelLaplace summation process. Notice that Camacho and Sad Theorem [8] guarantees the existence of at least one formal curve of Briot-Bouquet type.

Invariant curves of Briot-Bouquet type of $F$ are convergent when the infinitesimal generator of $F$ is convergent, but in general this is not the case. In fact, there are examples of diffeomorphisms in $\left(\mathbb{C}^{2}, 0\right)$ without analytic invariant curve (see 25). On the other hand, formal invariant curves that are not of Briot-Bouquet type appear in every saddle-node singularity of the infinitesimal generator whose weak direction (the one corresponding to the zero eigenvalue) is transversal to the exceptional divisor. For example, Euler's vector field has a purely formal invariant curve which is not of Briot-Bouquet type.

We prove Theorem 1 as a consequence of a more general result in arbitrary dimension $n$, which we explain briefly now.

One of our main tools is the reduction of the diffeomorphism to what we will call Ramis-Sibuya form along $\Gamma$ : assuming that $\Gamma$ is not contained in the set of fixed points of $F$, there is a process consisting in finitely many local blow-ups and ramifications, following the infinitely near points of the curve $\Gamma$, which transforms $F$ into a diffeomorphism which can be written as

$$
\widetilde{F}(x, \mathbf{y})=\left(x+x^{k+p+1}(1+o(1)), \mathbf{y}+x^{k}\left[\left(D(x)+x^{p} C\right) \mathbf{y}+O\left(x^{p+1}\right)\right]\right)
$$

where $(x, \mathbf{y}) \in \mathbb{C} \times \mathbb{C}^{n-1}, k \geq 1$ and either $p=0$ and $C \neq 0$ or $p \geq 1, D(x)$ is a diagonal matrix whose entries are polynomials of degree at most $p-1$ with $D(0) \neq 0$ and $C$ is a constant matrix which commutes with $D(x)$. Moreover, $\Gamma$ is transformed into a formal curve $\widetilde{\Gamma}$, invariant for $\widetilde{F}$, non-singular and transversal to the hypersurface $\{x=0\}$, the set of fixed points of $\widetilde{F}$. To the infinitesimal generator of a diffeomorphism $\widetilde{F}$ as in (1) we can associate a system of $n-1$ formal meromorphic ODEs which is, essentially, the starting point of Ramis-Sibuya's paper 24], and this is the reason for our name. In fact, we obtain a reduction of $F$ to Ramis-Sibuya form by transforming its infinitesimal generator $\log F$ into a vector field to which we can associate, by means of blow-ups and ramifications, a system as in Ramis-Sibuya paper, and whose exponential will be a diffeomorphism as in (11). In order for this approach to work, we need to prove first that blow-ups and ramifications are compatible with the operation of taking the infinitesimal generator. This compatibility, as well as the compatibility with the property of existence of parabolic curves asymptotic to $\Gamma$, is the content of section 5 .

In the two-dimensional case $n=2$, the reduction of a diffeomorphism $F$ to Ramis-Sibuya form is already established in the literature (see [1, 6]) and can be seen as a consequence of the well known result on reduction of singularities of vector fields (see for instance Seidenberg [27]).

In dimension $n \geq 3$, the reduction to Ramis-Sibuya form is not a completely unknown result either. In fact, after some initial blow-ups at infinitely near points of $\Gamma$ that transform the vector field $\log F$ into one to which we can associate a system of $n-1$ ODEs, the essential argument is an adaptation to the non-linear case of a well known result in the theory of systems of linear meromorphic ODEs, due to Turrittin [28. However, we have included a complete proof of the result in section 6] since we could not find any statement in the precise terms that we need for our purposes: for instance, we show that the centers of the blow-ups satisfy an additional property, besides the usual invariance, that guarantees the compatibility with the operation of 
taking the infinitesimal generator (see the definition of permissible center in section 5). Note that the infinitesimal generator of a diffeomorphism as in (11) has in particular a non-nilpotent linear part and hence a reduction to Ramis-Sibuya form contains a weak form (since we make use of ramifications) of a local uniformization of $\log F$ along $\Gamma$ (see [11, 22]).

Once we have reduction to Ramis-Sibuya form, it suffices to study the problem of existence of parabolic curves asymptotic to $\Gamma$ for diffeomorphisms $F$ as in (1). The case $p=0$ corresponds, in any dimension, to what we have called the Briot-Bouquet case in dimension two. In this case Écalle [14] and Hakim [17] prove that there exists at least one parabolic curve of $F$ asymptotic to $\Gamma$. We generalize their result to the case $p \geq 1$ (i.e. the eigenvalue corresponding to the tangent direction of $\Gamma$ vanishes) assuming an extra condition on $F$. When $p \geq 1$ put $D(x)=\operatorname{diag}\left(d_{2}(x), \ldots, d_{n}(x)\right)$ and define the saddle domain of $F$ (relatively to $\Gamma$ ) as the intersection of the "saddle domains" of all the linear ODEs $x^{p+1} y^{\prime}=d_{j}(x) y$, for $j=2, \ldots, n$. In section 3 we give details of this definition and we prove the following result (see Theorem [3.3): if the saddle domain is non-empty and contains one of the $(k+p)$-th roots of -1 then $F$ has a parabolic curve asymptotic to $\Gamma$. We obtain this parabolic curve as a fixed point of a contraction map in a convenient Banach space which, for the Briot-Bouquet case, coincides with the one used by Hakim in 17 .

Combining this result with the mentioned compatibility of the reduction to Ramis-Sibuya form with the existence of parabolic curves, we prove the following result. Given a diffeomorphism $F$ with an invariant formal curve $\Gamma$ not contained in the set of fixed points, we say that $\Gamma$ is well placed for $F$ if, after reduction to Ramis-Sibuya form (11), either we are in the Briot-Bouquet case $p=0$ or $p \geq 1$ and the saddle domain contains one of the $(k+p)$-th roots of -1 .

Theorem 2. Let $F \in \operatorname{Diff}_{1}\left(\mathbb{C}^{n}, 0\right)$ and let $\Gamma$ be an invariant formal curve of $F$ not contained in the set of fixed points of $F$. If $\Gamma$ is well placed for $F$, then there exists at least one attracting parabolic curve for $F$ asymptotic to $\Gamma$.

In the two-dimensional case $n=2$, we prove in section 4 that any formal invariant curve $\Gamma$ of $F$ is well placed either for $F$ or for the inverse diffeomorphism $F^{-1}$. Theorem 1 will then follow from Theorem 2 .

We conclude the introduction with a final remark. Theorem 2 establishes a sufficient condition on $F$ and $\Gamma$ in order that a parabolic curve of $F$ asymptotic to $\Gamma$ exists. To check such a condition we need to perform the process of reduction to Ramis-Sibuya form, which depends both on $F$ and on $\Gamma$. However, it is worth to mention that such a process only involves a finite part of the Taylor expansion of $F$ at the origin and hence the condition of $\Gamma$ being well placed for $F$ is finitely determined.

\section{Parabolic Curves and Formal invariant CuRVes}

Denote by $\operatorname{Diff}_{1}\left(\mathbb{C}^{n}, 0\right)$ the group of germs of holomorphic diffeomorphisms $F$ : $\left(\mathbb{C}^{n}, 0\right) \rightarrow\left(\mathbb{C}^{n}, 0\right)$ which are tangent to the identity, that is, $D F(0)=$ Id. Any $F \in \operatorname{Diff}_{1}\left(\mathbb{C}^{n}, 0\right)$ determines an endomorphism $\mathcal{F}: g \mapsto g \circ F$ of the local algebra $\mathcal{O}_{n}$ of germs of holomorphic functions at the origin. Conversely, any endomorphism of $\mathcal{O}_{n}$ inducing the identity in the cotangent space $\mathfrak{m} / \mathfrak{m}^{2}$, where $\mathfrak{m}$ is the maximal ideal, determines an element of $\operatorname{Diff}_{1}\left(\mathbb{C}^{n}, 0\right)$. Replacing $\mathcal{O}_{n}$ by its formal completion $\widehat{\mathcal{O}}_{n}$, we may analogously consider the group $\widehat{\operatorname{Diff}}_{1}\left(\mathbb{C}^{n}, 0\right)$ of formal tangent to the 
identity diffeomorphisms. If $F \in \widehat{\operatorname{Diff}}_{1}\left(\mathbb{C}^{n}, 0\right)$, its order is defined as the minimum $r$ such that $(\mathcal{F}-\mathrm{id})(\mathfrak{m}) \subset \mathfrak{m}^{r} \backslash \mathfrak{m}^{r+1}$.

To any formal tangent to the identity diffeomorphism $F$, we can associate its infinitesimal generator: the unique formal vector field $X \in \operatorname{Der}_{\mathbb{C}}\left(\widehat{\mathcal{O}}_{n}\right)$ which satisfies, for any $g \in \widehat{\mathcal{O}}_{n}$,

$$
\mathcal{F}(g)=g \circ F=\sum_{j=0}^{\infty} \frac{X^{j}(g)}{j !}
$$

where $X^{0}(g)=g$ and $X^{j}(g)=X\left(X^{j-1}(g)\right)$ for $j \geq 1$, or equivalently

$$
X(g)=\sum_{j=1}^{\infty} \frac{(-1)^{j+1}}{j}(\mathcal{F}-\mathrm{id})^{j}(g) .
$$

Its multiplicity at 0 , defined as $\nu_{0}(X)=\min \left\{l: X(\mathfrak{m}) \subset \mathfrak{m}^{l} \backslash \mathfrak{m}^{l+1}\right\}$, coincides with the order of $F$. Thus $\nu_{0}(X) \geq 2$. Reciprocally, each $X \in \operatorname{Der}_{\mathbb{C}}\left(\widehat{\mathcal{O}}_{n}\right)$ with $\nu_{0}(X) \geq 2$ is the infinitesimal generator of a unique formal diffeomorphism $F \in \widehat{\operatorname{Diff}}_{1}\left(\mathbb{C}^{n}, 0\right)$ satisfying (2). If $X$ is the infinitesimal generator of $F$, we write either $X=\log F$ or $F=\operatorname{Exp} X$. In general, the infinitesimal generator of $F \in \operatorname{Diff}_{1}\left(\mathbb{C}^{n}, 0\right)$ is not convergent (i.e. it does not belong to $\operatorname{Der}_{\mathbb{C}}\left(\mathcal{O}_{n}\right)$ ), although if $X$ is convergent then $\operatorname{Exp} X \in \operatorname{Diff}_{1}\left(\mathbb{C}^{n}, 0\right)$.

Once we choose coordinates $\mathbf{z}=\left(z_{1}, \ldots, z_{n}\right)$ at $0 \in \mathbb{C}^{n}$, a formal diffeomorphism $F \in \widehat{\operatorname{Diff}}_{1}\left(\mathbb{C}^{n}, 0\right)$ is written as $F(\mathbf{z})=\left(z_{1} \circ F, \ldots, z_{n} \circ F\right)=\left(z_{1}+f_{1}(\mathbf{z}), \ldots, z_{n}+f_{n}(\mathbf{z})\right)$, where $f_{j}(\mathbf{z}) \in \mathbb{C}[[\mathbf{z}]]$ and $\log F=a_{1}(\mathbf{z}) \frac{\partial}{\partial z_{1}}+\cdots+a_{n}(\mathbf{z}) \frac{\partial}{\partial z_{n}}$ with $a_{j}(\mathbf{z}) \in \mathbb{C}[[\mathbf{z}]]$. The order of $F$ is then the minimum of the orders of the series $f_{j}(\mathbf{z})$ for $j=1, \ldots, n$. Moreover, if $k=\nu_{0}(X)$, the homogeneous components of degree $k$ of the series $a_{j}(\mathbf{z})$ and of $f_{j}(\mathbf{z})$ are the same for $j=1, \ldots, n$. We also have, using identities (2) and (3), that the ideal of $\widehat{\mathcal{O}}_{n}$ generated by $\left\{f_{1}, f_{2}, \ldots, f_{n}\right\}$ is equal to the ideal generated by $\left\{a_{1}, a_{2}, \ldots, a_{n}\right\}$. Thus, in the case of $F$ being holomorphic, its set of fixed points $\operatorname{Fix}(F)$ coincides with the singular locus of $X$ (which is then an analytic set even when $X$ is formal).

A formal curve $\Gamma$ at $0 \in \mathbb{C}^{n}$ is by definition a prime ideal of the ring $\widehat{\mathcal{O}}_{n}$ such that the quotient ring $\widehat{\mathcal{O}}_{n} / \Gamma$ has dimension one. Once we fix analytic coordinates $\mathbf{z}$ at the origin, a formal curve $\Gamma$ is determined univocally by a formal parametrization: a tuple $\gamma(s)=\left(\gamma_{1}(s), \ldots, \gamma_{n}(s)\right) \in \mathbb{C}[[s]]^{n}$ so that $\gamma_{j}(0)=0$ and $g(\gamma(s)) \equiv 0$ if and only if $g \in \Gamma$. The multiplicity of $\Gamma$, denoted by $\nu_{0}(\Gamma)$, is the minimum of the orders of the components of any irreducible parametrization, i.e., a parametrization $\gamma(s)$ which cannot be written as $\gamma(s)=\sigma\left(s^{l}\right)$ with $\sigma(s)$ another parametrization of $\Gamma$ and $l>1$. The curve $\Gamma$ is called non-singular if $\nu_{0}(\Gamma)=1$. The tangent line of $\Gamma$ is, by definition, the complex line $T \Gamma$ at $0 \in \mathbb{C}^{n}$ whose projective coordinates are equal to $\left.\left[\gamma_{1}(s) / s^{m}, \ldots, \gamma_{n}(s) / s^{m}\right]\right|_{s=0}$ for $\gamma(s)$ an irreducible parametrization of $\Gamma$ and $m=\nu_{0}(\Gamma)$. For an appropriate choice of coordinates, we may always consider a parametrization of $\Gamma$ of the form $\gamma(s)=\left(s^{m}, \gamma_{2}(s), \ldots, \gamma_{n}(s)\right)$, where $m=\nu_{0}(\Gamma)$ and each $\gamma_{j}(s)$ has order at least $m$ (thus $T \Gamma$ is transversal to $\left\{z_{1}=0\right\}$ ). Such a parametrization is called a Puiseux parametrization of $\Gamma$.

Consider $F \in \operatorname{Diff}_{1}\left(\mathbb{C}^{n}, 0\right), X=\log F$ and let $\Gamma$ be a formal curve. We say that $\Gamma$ is invariant for $F$ if one of the following equivalent conditions holds:

1. $g \circ F \in \Gamma$ for any $g \in \Gamma$. 
2. $X(g) \in \Gamma$ for any $g \in \Gamma$.

3. For any parametrization $\gamma(s)$ of $\Gamma$ there exists $h(s) \in \mathbb{C}[[s]]$ such that $\left.X\right|_{\gamma(s)}=h(s) \gamma^{\prime}(s)$, where $\gamma^{\prime}(s) \in \mathbb{C}[[s]]^{n}$ is the vector of formal derivatives of the components of $\gamma(s)$.

The equivalence between the two first conditions follows from identities (2) and (3) (see 25] for a proof in dimension $n=2$ ). The equivalence between the two last ones is quite well known in differential algebra; a possible proof is the following: since the equivalence is preserved under blow-ups at infinitely near points of $\Gamma$, using reduction of singularities of $\Gamma$ we can assume that it is non-singular, and the equivalence is then easy to prove. Notice that in the last condition the series $h(s)$ is different from zero if and only if $\Gamma$ is not contained in the singular locus of $X$, which is equivalent to saying that $\Gamma$ is not contained in $\operatorname{Fix}(F)$. Observe also that, since $\log F^{-1}=-\log F$, a formal curve $\Gamma$ is invariant for $F$ if and only if it is invariant for $F^{-1}$.

It is worth to remark that, while a planar diffeomorphism $F \in \operatorname{Diff}_{1}\left(\mathbb{C}^{2}, 0\right)$ always has a formal invariant curve (by Camacho and Sad's Theorem [8]), this is not the case for $n>2$ (see the example of Gómez-Mont and Luengo [16]). On the other hand, formal invariant curves of diffeomorphisms, even in dimension two, do not need to be convergent (see for instance an example of Ribón [25]). A formal nonconvergent invariant curve $\Gamma$ has no geometric meaning a priori. We look for the possibility of finding a "geometric realization" of $\Gamma$ : roughly speaking, an analytic object attached to $\Gamma$ and invariant for $F$. A more precise statement of the problem is to find a parabolic curve of $F$ asymptotic to $\Gamma$.

Definition 2.1. An attracting parabolic curve for $F \in \operatorname{Diff}_{1}\left(\mathbb{C}^{n}, 0\right)$ is an injective holomorphic map $\varphi: \Delta \rightarrow \mathbb{C}^{n}$ where $\Delta$ is a simply connected domain in $\mathbb{C}$ with $0 \in \partial \Delta$ such that $\varphi(\Delta)$ is contained in the domain of a representant of $F$ and which satisfies:

1. $\varphi$ is continuous at the origin putting $\varphi(0)=0$ (continuity).

2. $F(\varphi(\Delta)) \subset \varphi(\Delta)$ (invariance).

3. $\lim _{j \rightarrow \infty} \varphi^{-1}\left(F^{j}(\varphi(s))\right)=0$ for all $s \in \Delta$ (stability).

$A$ repelling parabolic curve of $F$ is an attracting parabolic curve of the inverse diffeomorphism $F^{-1}$.

Definition 2.2. A parabolic curve $\varphi: \Delta \rightarrow \mathbb{C}^{n}$ will be called asymptotic to the formal curve $\Gamma$ if there exists a parametrization $\gamma(s) \in \mathbb{C}[[s]]^{n}$ of $\Gamma$ in some coordinates $\mathbf{z}$ of $\mathbb{C}^{n}$ such that $\mathbf{z} \circ \varphi$ has $\gamma(s)$ as asymptotic expansion at $s=0$ in $\Delta$; i.e., for any $N \in \mathbb{N}$, there exist constants $c_{N}, \varepsilon_{N}>0$ such that

$$
\left\|\mathbf{z} \circ \varphi(s)-J_{N} \gamma(s)\right\| \leq c_{N}|s|^{N+1} \forall s \in \Delta \text { with }|s| \leq \varepsilon_{N},
$$

where $J_{N} \gamma$ denotes the vector of truncations of the components of $\gamma$ up to order $N$, i.e. $\left\|\gamma(s)-J_{N} \gamma(s)\right\|=o\left(s^{N}\right)$.

A similar definition appears in [3] under the name "robust parabolic curve".

If the components of a parametrization of $\Gamma$ were multisummable (in the sense of Malgrange and Ramis [23, 21]), its multisum $\varphi: \Delta \rightarrow \mathbb{C}^{n}$, defined in some open sector $\Delta$, could be a good candidate to solve the problem of finding a parabolic curve asymptotic to $\Gamma$. However, as far as we know, multisummability of $\Gamma$ is an open question in general, except for the case where $\log F$ is a holomorphic vector 
field (see Braaksma [5]). We should also mention the work of López [19, where it is proved that $\Gamma$ is summable (and its sum gives a parabolic curve) in the case $n=2$ if $\Gamma$ is of Briot-Bouquet type (definition below), and the work of Brochero and López 7 , where the Gevrey character of the infinitesimal generator $\log F$ is proved, thus implying the Gevrey character of $\Gamma$ (see [9]).

Instead of searching for multisummability of $\Gamma$, we look for parabolic curves asymptotic to invariant formal curves by an approach similar to the one in Abate [1] and Brochero et al. [6]: we first analyze, in section 3, the case where $F$ can be written in a particular reduced form, called Ramis-Sibuya form, and then, in section 6, we prove that we can reduce $F$ to Ramis-Sibuya form by means of blow-ups and ramifications. Reduction to Ramis-Sibuya form in the case of two-dimensional diffeomorphisms is considerably easier and comes from the reduction of singularities of planar vector fields. We consider this case separately in section 4 where we prove Theorem 1

\section{Parabolic Curves For Diffeomorphisms in RAmis-Sibuya form}

Throughout this section, we consider $F \in \operatorname{Diff}_{1}\left(\mathbb{C}^{n}, 0\right)$ having a formal invariant non-singular curve $\Gamma$.

For convenience, a system of local analytic coordinates $\left(x, \mathbf{y}=\left(y_{2}, \ldots, y_{n}\right)\right)$ at $0 \in \mathbb{C}^{n}$ will be called adapted for $\Gamma$ if $\Gamma$ is transversal to the hypersurface $\{x=0\}$, thus having in those coordinates a parametrization as a graph $\gamma(x)=$ $\left(x, \gamma_{2}(x), \ldots, \gamma_{n}(x)\right) \in \mathbb{C}[[x]]^{n}$. The order of contact of $\Gamma$ with the $x$-axis is the minimum of the orders of the components $\gamma_{j}(x)$.

Definition 3.1. We say that the pair $(F, \Gamma)$ is in Ramis-Sibuya form (RS-form for short) if there exist adapted coordinates $(x, \mathbf{y})$ for $\Gamma$ at $0 \in \mathbb{C}^{n}$ for which $F$ is written as

$F(x, \mathbf{y})=\left(x+\lambda x^{k+p+1}(1+\psi(x, \mathbf{y})), \mathbf{y}+x^{k}\left[b(x)+\left(D(x)+x^{p} C+x^{p+1} A(x)\right) \mathbf{y}+O\left(\|\mathbf{y}\|^{2}\right)\right]\right)$,

where $k \geq 1, p \geq 0, \lambda \in \mathbb{C}^{*}, \psi \in \mathbb{C}\{x, \mathbf{y}\}$ with $\psi(0)=0, b(x) \in \mathbb{C}\{x\}^{n-1}$ with $b(0)=0, A(x)$ is a matrix with entries in $\mathbb{C}\{x\}$ and, moreover,

(i) either $p=0$ and $C \neq 0$ (this case is called Briot-Bouquet case) or

(ii) $p \geq 1, D(x)$ is a diagonal matrix of polynomials of degree at most $p-1$ with $D(0) \neq 0$ and $D(x)$ commutes with $C$.

Such adapted coordinates $(x, \mathbf{y})$ will be called RS-coordinates for $(F, \Gamma)$. The matrix $D(x)+x^{p} C$ is called the principal linear part of $(F, \Gamma)$ in the coordinates $(x, \mathbf{y})$.

Notice that both $k$ and $p$ do not depend on the choice of RS-coordinates. In fact $k+1$ is the order of the diffeomorphism $F$ and $k+p+1$ is the order of the restriction $\left.F\right|_{\Gamma} \in \widehat{\operatorname{Diff}}_{1}(\mathbb{C}, 0)$. The number $p$ is called the Poincaré rank of $(F, \Gamma)$. Notice also that the set of fixed points of $F$ is equal to $\{x=0\}$, since $\lambda \neq 0$, and then $\Gamma$ is not contained in $\operatorname{Fix}(F)$.

The infinitesimal generator of $F$ as in (4) is written as

$$
X=\log F=x^{k}\left[x^{p+1} u(x, \mathbf{y}) \frac{\partial}{\partial x}+\left(c(x)+\left(\mathcal{D}(x)+x^{p} \mathcal{C}+x^{p+1} \mathcal{A}(x)\right) \mathbf{y}+O\left(\|\mathbf{y}\|^{2}\right)\right) \frac{\partial}{\partial \mathbf{y}}\right]
$$

where $u(0,0)=\lambda, c(0)=0$ and either $p=0$ and $\mathcal{C} \neq 0$ or $p \geq 1$ and $\mathcal{D}(x)$ is a diagonal matrix of polynomials of degree at most $p-1$ which commutes with $\mathcal{C}$ 
and such that $\mathcal{D}(0) \neq 0$. Reciprocally, if $X$ is a vector field as in (5) satisfying the conditions above, then the diffeomorphism $\operatorname{Exp} X$ is in Ramis-Sibuya form. The matrix $\mathcal{D}(x)+x^{p} \mathcal{C}$ is called the principal linear part of $X$ in the coordinates $(x, \mathbf{y})$. To a vector field of the form (5) we can associate the system of $n-1$ formal ODEs

$$
x^{p+1} u(x, \mathbf{y}) \mathbf{y}^{\prime}=c(x)+\left(\mathcal{D}(x)+x^{p} \mathcal{C}+x^{p+1} \mathcal{A}(x)\right) \mathbf{y}+O\left(\|\mathbf{y}\|^{2}\right),
$$

which has irregular singular point at $x=0$ and Poincaré rank equal to $p$. The properties assumed for the principal linear part $\mathcal{D}(x)+x^{p} \mathcal{C}$ are essentially those considered in the work of Ramis and Sibuya [24, where multisummability of the formal solutions is proved in the case where the coefficients of the system are convergent. This is the reason of our choice of the name "Ramis-Sibuya form" in Definition 3.1

Remark 3.2. Assume that $(F, \Gamma)$ is in RS-form, written as in (44) in RS-coordinates $(x, \mathbf{y})$.

(a) Let $l$ denote the order of contact of $\Gamma$ with the $x$-axis. Then any component of the vector $b(x) \in \mathbb{C}\{x\}^{n-1}$ has order at least $l$.

(b) If $(\bar{x}, \overline{\mathbf{y}})$ are new coordinates at $0 \in \mathbb{C}^{n}$ obtained from $(x, \mathbf{y})$ by a change of variables of the form

$$
\bar{x}=h(x), \quad \overline{\mathbf{y}}=\mathbf{y}+\Lambda(x, \mathbf{y}), \text { where } h^{\prime}(0) \neq 0, \frac{\partial \Lambda}{\partial \mathbf{y}}(x, 0)=O\left(x^{p+1}\right)
$$

then $(\bar{x}, \overline{\mathbf{y}})$ are also RS-coordinates. Moreover, if $h(x)-x=O\left(x^{p+1}\right)$ then the principal linear parts of $(F, \Gamma)$ in the coordinates $(x, \mathbf{y})$ and $(\bar{x}, \overline{\mathbf{y}})$ are the same.

Now we deal with the problem of finding a parabolic curve of $F$ asymptotic to $\Gamma$ when $(F, \Gamma)$ is in RS-form. With the notations of Definition 3.1, we define the attracting directions of $(F, \Gamma)$ (with respect to the coordinates $(x, \mathbf{y})$ ) as the $k+p$ half-lines $\mathbb{R}_{+} \xi$, where $\xi^{k+p}=-\lambda$. If $\Gamma$ is convergent, the restriction $\left.F\right|_{\Gamma} \in$ $\operatorname{Diff}_{1}(\mathbb{C}, 0)$ has order $k+p+1$ and, by Leau and Fatou's theorem, any such direction is the bisectrix of an attracting petal of $\left.F\right|_{\Gamma}$, thus giving rise to $k+p$ attracting parabolic curves of $F$ defined in sectors bisected by these directions and asymptotic to $\Gamma$. When $\Gamma$ is formal, Écalle [14 and Hakim [17] obtain the same conclusion in the Briot-Bouquet case. Here, we extend this result to the non Briot-Bouquet case $(p \geq 1)$, proving the existence of a parabolic curve attached to a fixed attracting direction $\tau$ if some extra hypothesis (concerning $\tau$ ) is satisfied. Our arguments are based mainly on Hakim's paper; however, since our statement is not exactly the same as Hakim's one, our result is stated for $(F, \Gamma)$ irrespective of the value of the Poincaré rank $p$.

Let $D(x)+x^{p} C$ be the principal linear part of $(F, \Gamma)$ with respect to coordinates $(x, \mathbf{y})$. Write $D(x)=\operatorname{diag}\left(d_{2}(x), \ldots, d_{n}(x)\right)$ where each $d_{j}(x)$ is a polynomial of degree at most $p-1$. For each $j$ such that $d_{j}(x) \not \equiv 0$, we denote by $\nu_{j}$ the order of $d_{j}$ at 0 and write $d_{j}(x)=d_{j 0} x^{\nu_{j}}+\cdots$ with $d_{j 0} \neq 0$. Observe that if $p \geq 1$ then $\nu_{j}=0$ for at least one $j$, since $D(0) \neq 0$. Notice also that if $\mathcal{D}(x)+x^{p} \mathcal{C}$ is the principal linear part of $\log F$ then the $j$-th entry of $\mathcal{D}(x)$ is also of the form $d_{j 0} x^{\nu_{j}}+\cdots$ if $d_{j}(x) \not \equiv 0$, and is equal to zero if $d_{j}(x) \equiv 0$.

Let us define the saddle domain of $(F, \Gamma)$ (in the coordinates $(x, \mathbf{y})$ ) as the set

$$
V=\bigcap_{\substack{2 \leq j \leq n \\ d_{j} \neq 0}}\left\{x \in \mathbb{C}: \operatorname{Re}\left(-\frac{d_{j 0}}{\lambda x^{p-\nu_{j}}}\right)>0\right\} \subset \mathbb{C} .
$$


We notice that the saddle domain $V$ is the whole plane $\mathbb{C}$ in the Briot-Bouquet case $p=0$, and a union of proper open sectors at the origin in the case $p \geq 1$. Observe that $V$ is non-empty if $n=2$. Also, if $p \geq 1$ and $D(0)$ is invertible then $V \neq \emptyset$ implies that the eigenvalues of $D(0)$ belong to the so called "Poincaré domain", i.e. their convex hull in the plane does not contain 0 . The name "saddle domain" comes from the following fact: if $p \geq 1$, the linear system $x^{p+1} Y^{\prime}=\overline{\mathcal{D}}(x) Y$ of ODEs, where $\overline{\mathcal{D}}(x)$ is the diagonal submatrix of $\mathcal{D}(x)$ formed by its nonzero entries, has $Y=0$ as the unique solution which is bounded in closed subsectors of $V$, while the other ones are unbounded along any direction contained in $V$.

We are now ready to state the main result in this section. We use the standard notation $S(\tau, \alpha, r)$ for the open sector at $0 \in \mathbb{C}$ bisected by $\tau$ with opening $\alpha>0$ and radius $r \in \mathbb{R}^{+} \cup\{\infty\}$.

Theorem 3.3. Assume that $F \in \operatorname{Diff}_{1}\left(\mathbb{C}^{n}, 0\right)$ has a formal invariant curve $\Gamma$ such that $(F, \Gamma)$ is in Ramis-Sibuya form. Let $\tau$ be an attracting direction and let $V$ be the saddle domain in some $R S$-coordinates $(x, \mathbf{y})$. If $\tau$ is contained in $V$ then there exists an attracting parabolic curve for $F$ asymptotic to $\Gamma$. More precisely, there exist positive numbers $\eta_{0}, \delta_{0}$ with $S\left(\tau, \eta_{0}, \delta_{0}\right) \subset V$ and a holomorphic map $\bar{\varphi}: S\left(\tau, \eta_{0}, \delta_{0}\right) \rightarrow \mathbb{C}^{n-1}$ such that

(i) The map $\varphi: S\left(\tau, \eta_{0}, \delta_{0}\right) \rightarrow \mathbb{C}^{n}$ given by $x \mapsto(x, \bar{\varphi}(x))$ in the coordinates $(x, \mathbf{y})$ is an attracting parabolic curve for $F$ asymptotic to $\Gamma$.

(ii) For any $0<\eta \leq \eta_{0}$ there exists $0<\delta \leq \delta_{0}$ such that, if $\delta^{\prime} \leq \delta$, then the restriction of $\varphi$ to $S\left(\tau, \eta, \delta^{\prime}\right)$ is also an attracting parabolic curve for $F$.

(iii) If $W$ is any sector containing the direction $\tau$ and $\psi: W \rightarrow \mathbb{C}^{n}$ is an attracting parabolic curve of $F$ asymptotic to $\Gamma$, defined by $\psi(x)=(x, \bar{\psi}(x))$ in the coordinates $(x, \mathbf{y})$, then $\varphi \equiv \psi$ in $S\left(\tau, \eta_{0}, \delta_{0}\right) \cap W$.

Remark 3.4. Although both the attracting direction $\tau$ and the saddle domain $V$ depend on the RS-coordinates, the condition $\tau \subset V$ is independent of the coordinates.

The rest of this section is devoted to the proof of Theorem 3.3 .

Choosing coordinates.- We start with an expression of $F$ as in (4) in the given coordinates $(x, \mathbf{y})$. Up to a linear change of the form $x \mapsto \alpha x$, we can assume that $\lambda=-1$. Moreover, it is a routine to check that a polynomial change of variables of the form $x \mapsto x+P(x)$ with $P(x)=O\left(x^{p+1}\right)$ permits to consider new RS-coordinates $(x, \mathbf{y})$ for which

$$
x \circ F(x, \mathbf{y})=x-x^{k+p+1}+O\left(x^{k+p+1}\|\mathbf{y}\|, x^{k+2 p+2}\right) .
$$

Notice, using Remark 3.2 that the principal linear part has not changed. We will need to consider coordinates having high contact with $\Gamma$. In fact, consider a Puiseux parametrization $\gamma(x)=(x, \bar{\gamma}(x)) \in \mathbb{C}[[x]]^{n}$ of $\Gamma$ in the coordinates $(x, \mathbf{y})$. For any $m \geq 2$, we consider new coordinates $\left(x, \mathbf{y}_{m}\right)$ with $\mathbf{y}_{m}=\mathbf{y}-J_{m+p-1} \bar{\gamma}(x)$ (recall that $J_{N} \bar{\gamma}(x)$ denotes the truncation of $\bar{\gamma}(x)$ up to order $\left.N\right)$. Then $\left(x, \mathbf{y}_{m}\right)$ are new RS-coordinates for which $\Gamma$ has order of contact at least $m+p$ with the $x$-axis. Hence, using Remark 3.2, $F$ is written as

(6)

$$
\begin{aligned}
x \circ F\left(x, \mathbf{y}_{m}\right) & =x-x^{k+p+1}+O\left(x^{k+p+1}\left\|\mathbf{y}_{m}\right\|, x^{k+2 p+2}\right) \\
\mathbf{y}_{m} \circ F\left(x, \mathbf{y}_{m}\right) & =\mathbf{y}_{m}+x^{k}\left(D(x)+x^{p} C\right) \mathbf{y}_{m}+O\left(x^{k+p+1}\left\|\mathbf{y}_{m}\right\|, x^{k}\left\|\mathbf{y}_{m}\right\|^{2}, x^{k+p+m}\right)
\end{aligned}
$$


Notice that the principal linear part of $(F, \Gamma)$ with respect to $\left(x, \mathbf{y}_{m}\right)$ does not depend on $m$. Neither do the attracting directions and the saddle domain $V$, since the coordinate $x$ is preserved. Write, as above, $D(x)=\operatorname{diag}\left(d_{2}(x), \ldots, d_{n}(x)\right)$ and $d_{j}(x)=d_{j 0} x^{\nu_{j}}+\cdots$ with $d_{j 0} \neq 0$ if $d_{j}(x) \not \equiv 0$.

Reduction to parabolic curves having $m$-th order contact with $\Gamma$.- Given an integer $m \geq p+2$ and constants $\eta, \delta>0$, we consider the Banach space $\left(\mathcal{B}_{\eta, \delta}^{m}, \mathbf{n}\right)$ defined by

$$
\mathcal{B}_{\eta, \delta}^{m}=\left\{u \in \mathcal{O}\left(S(\tau, \eta, \delta), \mathbb{C}^{n-1}\right): \mathbf{n}(u)=\sup \left\{\frac{\|u(x)\|}{|x|^{m-1}}: x \in S(\tau, \eta, \delta)\right\}<\infty\right\}
$$

and the closed subspace

$$
\mathcal{H}_{\eta, \delta}^{m}=\left\{u \in \mathcal{B}_{\eta, \delta}^{m}: \mathbf{n}(u) \leq 1,\left\|u^{\prime}(x)\right\| \leq|x|^{m-p-2} \forall x \in S(\tau, \eta, \delta)\right\} .
$$

Let $I$ be the interval of points $\eta \in \mathbb{R}$ satisfying $0<\eta<2 \pi /(k+p)$ and

$$
S(\tau, \eta, \infty) \subset V \cap \bigcap_{\substack{2 \leq j \leq n \\ d_{j} \neq 0}}\left\{x \in \mathbb{C}: \operatorname{Re}\left(d_{j 0} x^{k+\nu_{j}}\right)>0\right\} .
$$

Notice that $I \neq \emptyset$ by the hypothesis on the attracting direction $\tau$.

Fix an integer $m_{0}$ such that $m_{0} \geq \max \left\{p+2, p+2-\min \left\{\operatorname{Re}\left(\alpha_{j}\right): \alpha_{j} \in\right.\right.$ $\operatorname{Spec}(\mathcal{C})\}\}$, where $\mathcal{D}(x)+x^{p} \mathcal{C}$ is the principal linear part of the infinitesimal generator of $F$, with the notations of (5).

Proposition 3.5. For any $m \geq m_{0}$ and any $\eta \in I$ there exists $\delta=\delta(m, \eta)>0$, with $S(\tau, \eta, \delta)$ contained in the domain of the coordinates $\left(x, \mathbf{y}_{m}\right)$, and for any $\delta^{\prime}$ with $0<\delta^{\prime} \leq \delta$ there exists a unique $u_{m, \delta^{\prime}} \in \mathcal{H}_{\eta, \delta^{\prime}}^{m}$ such that:

(a) The map $\varphi_{m, \delta^{\prime}}: S\left(\tau, \eta, \delta^{\prime}\right) \rightarrow \mathbb{C}^{n}$ written in the coordinates $\left(x, \mathbf{y}_{m}\right)$ as $\varphi_{m, \delta^{\prime}}$ : $x \mapsto\left(x, u_{m, \delta^{\prime}}(x)\right)$ is an attracting parabolic curve for $F$.

(b) For any $0<\delta^{\prime} \leq \delta$, we have $u_{m, \delta^{\prime}}=\left.u_{m, \delta}\right|_{S\left(\tau, \eta, \delta^{\prime}\right)}$.

(c) If $W$ is a sector containing $S\left(\tau, \eta, \delta^{\prime}\right)$ for some $\delta^{\prime} \leq \delta$ and $\psi: W \rightarrow \mathbb{C}^{n}$ is an attracting parabolic curve for $F$ written in coordinates $\left(x, \mathbf{y}_{m}\right)$ as $\psi(x)=$ $(x, v(x))$, with $\left.v\right|_{S\left(\tau, \eta, \delta^{\prime}\right)} \in \mathcal{H}_{\eta, \delta^{\prime}}^{m}$, then $u_{m, \delta} \equiv v$ in $S\left(\tau, \eta, \delta^{\prime}\right)$.

Before the proof of Proposition 3.5 let us see how it permits to prove Theorem 3.3 Apply Proposition 3.5 to $m^{\prime}>m \geq m_{0}$ and a fixed constant $\eta_{0} \in I$. We obtain two parabolic curves $\varphi_{m}: S\left(\tau, \eta_{0}, \delta_{m}\right) \rightarrow \mathbb{C}^{n}, \varphi_{m^{\prime}}: S\left(\tau, \eta_{0}, \delta_{m^{\prime}}\right) \rightarrow \mathbb{C}^{n}$ of $F$ which we write in coordinates $\left(x, \mathbf{y}_{m}\right)$ and $\left(x, \mathbf{y}_{m^{\prime}}\right)$ respectively as $\varphi_{m}(x)=\left(x, u_{m}(x)\right)$, with $u_{m} \in \mathcal{H}_{\eta_{0}, \delta_{m}}^{m}$, and $\varphi_{m^{\prime}}(x)=\left(x, u_{m^{\prime}}(x)\right)$, with $u_{m^{\prime}} \in \mathcal{H}_{\eta_{0}, \delta_{m^{\prime}}}^{m^{\prime}}$. Let $\rho=\min \left\{\delta_{m}, \delta_{m^{\prime}}\right\}$ and write the restriction of $\varphi_{m^{\prime}}$ to $S\left(\tau, \eta_{0}, \rho\right)$ in the coordinates $\left(x, \mathbf{y}_{m}\right)$ as $\varphi_{m^{\prime}}(x)=$ $(x, v(x))$. Then we have that $v(x)=P(x)+u_{m^{\prime}}(x)$, where $P(x)=J_{m^{\prime}+p-1} \bar{\gamma}(x)-$ $J_{m+p-1} \bar{\gamma}(x)$, a vector of polynomials of order at least $m+p$ and of degree at most $m^{\prime}+p-1$. We obtain that the restriction of $v$ to $S\left(\tau, \eta_{0}, \rho^{\prime}\right)$ belongs to $\mathcal{H}_{\eta_{0}, \rho^{\prime}}^{m}$ for some $0<\rho^{\prime} \leq \rho$ and, by Proposition 3.5. (c), we have $v \equiv u_{m}$ in $S\left(\tau, \eta_{0}, \rho^{\prime}\right)$ (notice that $\left.\varphi_{m^{\prime}}\right|_{S\left(\tau, \eta_{0}, \rho^{\prime}\right)}$ is again a parabolic curve by part (b)). Thus $\varphi_{m}$ and $\varphi_{m^{\prime}}$ coincide in their common domains and we obtain a holomorphic map

$$
\varphi: \bigcup_{m \geq m_{0}} S\left(\tau, \eta_{0}, \delta_{m}\right) \rightarrow \mathbb{C}^{n} .
$$


We may assume that $\delta_{m} \leq \delta_{m_{0}}$ for any $m \geq m_{0}$ and thus $\varphi$ is defined in $S\left(\tau, \eta_{0}, \delta_{0}\right)$ where $\delta_{0}=\delta_{m_{0}}$. By construction, $\varphi$ is a parabolic curve of $F$ asymptotic to $\Gamma$, proving part (i) of Theorem 3.3. Part (ii) follows from properties (b) and (c) of Proposition 3.5 stated for $\eta \leq \eta_{0}$. Finally, let us show property (iii) of Theorem 3.3 The map $\bar{\psi}_{m_{0}}(x)=\bar{\psi}(x)-\bar{J}_{m_{0}+p-1} \bar{\gamma}(x)$ has the formal series $\bar{\gamma}(x)-J_{m_{0}+p-1} \bar{\gamma}(x)$ as asymptotic expansion on $W$ at 0 . Since this series has order at least $m_{0}+p$, we deduce that there exist some $\eta, \delta>0$ such that $S(\tau, \eta, \delta) \subset W$ and the restriction $\left.\bar{\psi}_{m_{0}}\right|_{S(\tau, \eta, \delta)}$ belongs to $\mathcal{H}_{\eta, \delta}^{m_{0}}$. On the other hand, $\left(x, \bar{\psi}_{m_{0}}(x)\right)$ is the expression of $\psi$ in the coordinates $\left(x, \mathbf{y}_{m_{0}}\right)$ and hence, by Proposition 3.5. (b) and (c), up to considering smaller constants $\eta, \delta$, we obtain that $\psi$ coincides with $\varphi$ on $S(\tau, \eta, \delta)$ and thus on $W \cap S\left(\tau, \eta_{0}, \delta_{0}\right)$.

The rest of the paragraph is devoted to the proof of Proposition 3.5 divided in several steps. We fix some $m \geq m_{0}$ and $\eta \in I$ and assume that $F$ is written as in (6) in the coordinates $\left(x, \mathbf{y}_{m}\right)$. For simplicity, from now on we will drop the indexes " $m, \eta$ ". In particular $\mathbf{y}=\mathbf{y}_{m}, \mathcal{B}_{\delta}=\mathcal{B}_{\eta, \delta}^{m}, \mathcal{H}_{\delta}=\mathcal{H}_{\eta, \delta}^{m}$ and $S_{\delta}=S(\tau, \eta, \delta)$.

Denote by $f(x, \mathbf{y})=x \circ F, \bar{F}(x, \mathbf{y})=\mathbf{y} \circ F$ the series defined in (6) and let $\varepsilon>0$ be small enough so that these series are convergent for any $(x, \mathbf{y})$ with $|x| \leq \varepsilon$, $\|\mathbf{y}\| \leq \varepsilon^{m-1}$. We look first for an element $u \in \mathcal{H}_{\delta}$, with $\delta$ sufficiently small, so that

$$
u(f(x, u(x)))=\bar{F}(x, u(x))
$$

Such an $u$ will be constructed as a fixed point of a contracting map in $\mathcal{H}_{\delta}$, in an analogous way to what is done in Hakim's work [17] for the Briot-Bouquet case.

Some technical results.- Let $D(x)+x^{p} C$ and $\mathcal{D}(x)+x^{p} \mathcal{C}$ be respectively the principal linear parts of the diffeomorphism $F$ and of its infinitesimal generator $X=\log F$ in the RS-coordinates $(x, \mathbf{y})$ (with the notations of (6) and (5) ). Recall that they do not depend on $m$. We have that

$$
I+x^{k}\left(D(x)+x^{p} C\right)=J_{k+p}\left(\exp \left(x^{k}\left(\mathcal{D}(x)+x^{p} \mathcal{C}\right)\right)\right) .
$$

Define

$$
\widehat{E}(x)=\exp \left(-\int \frac{\mathcal{D}(x)}{x^{p+1}} d x\right) ; E(x)=\widehat{E}(x) x^{-\mathcal{C}} .
$$

Observe that $\widehat{E}(x)$ is a fundamental solution of the linear system of ODEs $x^{p+1} Y^{\prime}=-\mathcal{D}(x) Y$, since $\mathcal{D}(x)$ is diagonal, whereas $E(x)$ is a fundamental solution of $x^{p+1} Y^{\prime}=-\left(\mathcal{D}(x)+x^{p} \mathcal{C}\right) Y$, since $\mathcal{D}(x)$ commutes with $\mathcal{C}$. The matrix $\widehat{E}(x)$ has an essential singularity at $x=0$ when $p \geq 1$ and $E(x)$ is a multivalued function of $x \in \mathbb{C}^{*}$. They have the interesting properties stated in the following lemma, inspired by Lemma 3.1 in [26]:

Lemma 3.6. For any $(x, \mathbf{y})$ sufficiently small and $x \neq 0$, we have

$$
\begin{aligned}
& \widehat{E}(x) \widehat{E}(f(x, \mathbf{y}))^{-1}=\exp \left(-x^{k} \mathcal{D}(x)\right)+O\left(x^{k+p+1}, x^{k}\|\mathbf{y}\|\right) \\
& E(x) E(f(x, \mathbf{y}))^{-1}=\exp \left(-x^{k}\left(\mathcal{D}(x)+x^{p} \mathcal{C}\right)\right)+O\left(x^{k+p+1}, x^{k}\|\mathbf{y}\|\right)
\end{aligned}
$$

Proof. Let $Z(x)$ be either $E(x)$ or $\widehat{E}(x)$, a fundamental solution of the system $x^{p+1} Y^{\prime}=-B(x) Y$, where $B(x)$ is either $\mathcal{D}(x)+x^{p} \mathcal{C}$ or $\mathcal{D}(x)$, correspondingly. Put $\Omega(x, z)=Z\left(x+x^{p+1} z\right)$. If we fix $x$ and consider $\Omega$ as a function of $z$ then it satisfies 
the (regular) system

$$
\frac{\partial \Omega}{\partial z}=\frac{-B\left(x+x^{p+1} z\right)}{\left(1+x^{p} z\right)^{p+1}} \Omega(x, z) .
$$

On the other hand, we have $\Omega(x, 0)=Z(x)$ and thus

$$
\Omega(x, z)=\exp \left(-\int_{0}^{z} \frac{B\left(x+x^{p+1} t\right)}{\left(1+x^{p} t\right)^{p+1}} d t\right) Z(x)
$$

(using again that $\mathcal{D}(x)$ is diagonal and commutes with $\mathcal{C}$ ). Hence

$$
Z(x) Z\left(x+x^{p+1} z\right)^{-1}=Z(x) \Omega(x, z)^{-1}=\exp \left(\int_{0}^{z} \frac{B\left(x+x^{p+1} t\right)}{\left(1+x^{p} t\right)^{p+1}} d t\right) .
$$

The integrand in the equation above is an analytic function of $(x, t)$ and may be written as $\frac{B\left(x+x^{p+1} t\right)}{\left(1+x^{p} t\right)^{p+1}}=B(x)+O\left(x^{p} t\right)$. By integration, we obtain, for any $x \neq 0$ and $z$ sufficiently small,

$$
Z(x) Z\left(x+x^{p+1} z\right)^{-1}=\exp (z B(x))+x^{p} z^{2} \Lambda(x, z),
$$

where $\Lambda$ is analytic at the origin. The result follows using the expression of $f(x, \mathbf{y})$.

Dynamics in dimension one.- Given $u \in \mathcal{B}_{\varepsilon}$ with $\mathbf{n}(u) \leq 1$, we consider the holomorphic map $f_{u}: S_{\varepsilon} \rightarrow \mathbb{C}$ defined by $f_{u}(x)=f(x, u(x))$.

Notice (since $m \geq p+2$ ) that $\left|f_{u}(x)-x+x^{k+p+1}\right| \leq B|x|^{k+2 p+2}$ for $x \in S_{\varepsilon}$, with some $B>0$ independent of $u$. Then, taking into account that $\eta<2 \pi /(k+p)$ and arguing as in the proof of Leau and Fatou flower theorem in 20, we can prove the following result.

Proposition 3.7. There exists a constant $\delta$ with $0<\delta \leq \varepsilon$ such that, for every $0<\delta^{\prime} \leq \delta$ and every $u \in \mathcal{H}_{\delta^{\prime}}$ we have $f_{u}\left(S_{\delta^{\prime}}\right) \subset S_{\delta^{\prime}}$. Moreover, there is a constant $c>0$ such that, if $x_{0} \in S_{\delta}$ and we denote by $x_{j}=f_{u}\left(x_{j-1}\right)$, for $j \geq 1$, the orbit of $x_{0}$ by $f_{u}$, then for all $j \in \mathbb{N}$ we have

$$
x_{j}^{k+p} \sim \frac{1}{(k+p) j} \quad \text { and } \quad\left|x_{j}\right|^{k+p} \leq c \frac{\left|x_{0}\right|^{k+p}}{1+j\left|x_{0}\right|^{k+p}}
$$

(where $w_{j} \sim z_{j}$ means that $\lim _{j \rightarrow \infty} w_{j} / z_{j}=1$ ).

We also need the following lemma.

Lemma 3.8. Put $\sigma=\min \left\{\operatorname{Re}\left(\alpha_{j}\right): \alpha_{j} \in \operatorname{Spec}(\mathcal{C})\right\}$. With the same notations as in Proposition 3.7, and if $\delta$ is sufficiently small, we have:

(i) For any real number $s>k+p$, there exists a constant $K_{s}>0$ such that $\sum_{j \geq 0}\left|x_{j}\right|^{s} \leq K_{s}\left|x_{0}\right|^{s-k-p}$.

(ii) There exist constants $M_{1}, M_{2}>0$ such that $\left\|\left(x_{j} / x_{0}\right)^{\mathcal{C}}\right\| \leq M_{1}\left|x_{j} / x_{0}\right|^{\sigma-1 / 2}$ and $\left\|\widehat{E}\left(x_{0}\right) \widehat{E}\left(x_{j}\right)^{-1}\right\| \leq M_{2}$ for any $x_{0} \in S_{\delta}$ and any $j \geq 0$.

Proof. Part (i) is proved without difficulty using the second inequality in Proposition 3.7 (see [17]). For the first inequality of (ii), assuming $\mathcal{C}=U+N$ is in Jordan form ( $U$ diagonal and $N$ nilpotent), there exists a constant $M$, depending only on $\mathcal{C}$, such that

$$
\left\|z^{\mathcal{C}}\right\| \leq M|z|^{-1 / 2}\left\|z^{U}\right\| \leq M|z|^{\sigma-1 / 2}, \text { for } 0<|z| \leq 1 .
$$


The result follows putting $z=\frac{1}{c^{1 /(k+p)}} \frac{x_{j}}{x_{0}}$, where $c$ is the constant of Proposition 3.7 Let us prove the second inequality of (ii). Using Lemma 3.6. the definition of $\mathcal{H}_{\delta}$ and the fact that $m \geq p+2$, we may write

$$
\widehat{E}\left(x_{0}\right) \widehat{E}\left(x_{1}\right)^{-1}=\exp \left(-x_{0}^{k} \mathcal{D}\left(x_{0}\right)\right)+\theta_{u}\left(x_{0}\right)
$$

where $\left\|\theta_{u}\left(x_{0}\right)\right\| \leq K\left|x_{0}\right|^{k+p+1}$ for any $x_{0} \in S_{\delta}$ and any $u \in \mathcal{H}_{\delta}$, with some $K>0$ independent of $u$. From equation (8), we have $\mathcal{D}(x)=\operatorname{diag}\left(b_{2}(x), \ldots, b_{n}(x)\right)$ where each $b_{i}(x) \in \mathbb{C}[x]$ has degree at most $p-1$ and satisfies $b_{i}(x)=d_{i 0} x^{\nu_{i}}+O\left(x^{\nu_{i}+1}\right)$ if $d_{i} \not \equiv 0$ or $b_{i} \equiv 0$ otherwise. By the definition of the interval $I, \operatorname{Re}\left(d_{i 0} x_{l}^{k+\nu_{i}}\right)>0$ for all $l \geq 0$ if $b_{i}(x) \not \equiv 0$ (recall that $x_{l} \in S_{\delta}$ for all $l \geq 0$ ). We obtain

$$
\left\|\widehat{E}\left(x_{0}\right) \widehat{E}\left(x_{j}\right)^{-1}\right\| \leq \prod_{l=0}^{j-1}\left(1+K\left|x_{l}\right|^{k+p+1}\right) \leq \prod_{l=0}^{\infty}\left(1+K\left|x_{l}\right|^{k+p+1}\right)
$$

for $\delta$ sufficiently small. The result follows from the fact that $x_{j}^{k+p} \sim \frac{1}{(k+p) j}$ and a classical criterium of convergence of infinite products.

The contraction map.- Define $H(x, \mathbf{y})=\mathbf{y}-E(x) E(f(x, \mathbf{y}))^{-1} \bar{F}(x, \mathbf{y}) \in \mathbb{C}\{x, \mathbf{y}\}$. Using Lemma 3.6 and identity (8), we have

$$
H(x, \mathbf{y})=O\left(x^{k+p+1}\|\mathbf{y}\|, x^{k}\|\mathbf{y}\|^{2}, x^{k+p+m}\right) .
$$

Proposition 3.9. If $\delta>0$ is sufficiently small and we put $x_{j}=f_{u}\left(x_{j-1}\right)$ for $j \geq 1$ for $u \in \mathcal{H}_{\delta}$ and $x_{0} \in S_{\delta}$, the series

$$
T u\left(x_{0}\right)=\sum_{j \geq 0} E\left(x_{0}\right) E\left(x_{j}\right)^{-1} H\left(x_{j}, u\left(x_{j}\right)\right)
$$

is normally convergent and defines a contracting map $T: u \mapsto T u$ from $\mathcal{H}_{\delta}$ to itself. Moreover, $u \in \mathcal{H}_{\delta}$ is a fixed point of $T$ if and only if $u$ satisfies equation (7).

Remark 3.10. In the Briot-Bouquet case $p=0$ we have from (8) that $\mathcal{D}(x) \equiv 0$ and $\mathcal{C}=C$, so the operator $T$ and the space $\mathcal{H}_{\delta}$ are exactly the ones considered by Hakim in [17.

Proof. By (9), there exists a constant $K>0$ such that, if $\delta$ is small enough and $x \in S_{\delta}$ then $\|H(x, u(x))\| \leq K|x|^{k+p+m}$ independently of $u \in \mathcal{H}_{\delta}$ (recall that we have assumed that $m \geq p+2$ ). Thus, using Lemma 3.8 (ii),

$$
\left\|E\left(x_{0}\right) E\left(x_{j}\right)^{-1} H\left(x_{j}, u\left(x_{j}\right)\right)\right\| \leq M_{1} M_{2} K \frac{\left|x_{j}\right|^{\sigma-\frac{1}{2}+k+p+m}}{\left|x_{0}\right|^{\sigma-\frac{1}{2}}}
$$

and using Lemma 3.8 , (i), the series $T u\left(x_{0}\right)$ is normally convergent for any $x_{0} \in S_{\delta}$ and $u \in \mathcal{H}_{\delta}$, defines a holomorphic map $T u \in \mathcal{O}\left(S_{\delta}, \mathbb{C}^{n-1}\right)$ and satisfies $T u(x)=$ $O\left(|x|^{m}\right)$. Then, $\|T u(x)\| \leq|x|^{m-1}$ for $|x|<\delta$, if $\delta$ is small enough. We compute 
the derivative:

$$
\begin{aligned}
(T u)^{\prime}\left(x_{0}\right) & =\sum_{j \geq 0} E^{\prime}\left(x_{0}\right) E\left(x_{j}\right)^{-1} H\left(x_{j}, u\left(x_{j}\right)\right) \\
& -\sum_{j \geq 0} E\left(x_{0}\right) E\left(x_{j}\right)^{-1} x_{j}^{\prime} E^{\prime}\left(x_{j}\right) E\left(x_{j}\right)^{-1} H\left(x_{j}, u\left(x_{j}\right)\right) \\
& +\sum_{j \geq 0} E\left(x_{0}\right) E\left(x_{j}\right)^{-1} x_{j}^{\prime} \frac{\partial H}{\partial x}\left(x_{j}, u\left(x_{j}\right)\right) \\
& +\sum_{j \geq 0} E\left(x_{0}\right) E\left(x_{j}\right)^{-1} x_{j}^{\prime} \frac{\partial H}{\partial \mathbf{y}}\left(x_{j}, u\left(x_{j}\right)\right) u^{\prime}\left(x_{j}\right) .
\end{aligned}
$$

where the notation $x_{j}^{\prime}$ stands for the derivative, at the point $x_{0}$, of $f_{u}^{j}(x)$ as a function of $x$. Arguing as in [17, Lemma 4.4, we have that $\left|x_{j}^{\prime}\right| \leq 2\left|x_{j} / x_{0}\right|^{k+p+1}$ for all $j \geq 0$. On the other hand, recall that $E^{\prime}(x)=-\frac{\mathcal{D}(x)+\mathcal{C} x^{p}}{x^{p+1}} E(x)$. Using these two arguments, equations (9), (10), Lemma 3.8 and the inequality $\left\|u^{\prime}\left(x_{j}\right)\right\| \leq\left|x_{j}\right|^{m-p-2}$ from the definition of $\mathcal{H}_{\delta}$, it is easy (but tedious) to check that each of the four summands in the expression of $(T u)^{\prime}\left(x_{0}\right)$ is bounded by a positive constant times $\left|x_{0}\right|^{m-p-1}$. Therefore, $\left\|(T u)^{\prime}(x)\right\| \leq|x|^{m-p-2}$ for $|x|<\delta$, if $\delta$ is small enough, which shows that $T u \in \mathcal{H}_{\delta}$.

Let us prove that $T$ is a contraction map. Take $u, v \in \mathcal{H}_{\delta}$. For $x_{0} \in S_{\delta}$, put $x_{j}=f_{u}^{j}\left(x_{0}\right)$ and $y_{j}=f_{v}^{j}\left(x_{0}\right)$. Arguing as in [17, Lemma 4.9, with a slight modification as in [19], Lemma 4.4, if $\delta$ is small enough, there exists $M>0$, independent of $x_{0} \in S_{\delta}$ and $u, v \in \mathcal{H}_{\delta}$, such that

$$
\left|y_{j}-x_{j}\right| \leq M\left|x_{0}\right|^{m} \mathbf{n}(v-u) .
$$

Write $T u\left(x_{0}\right)-T v\left(x_{0}\right)=U_{1}+U_{2}+U_{3}$, where

$$
\begin{aligned}
U_{1} & =\sum_{j \geq 0} E\left(x_{0}\right) E\left(x_{j}\right)^{-1}\left[H\left(x_{j}, u\left(x_{j}\right)\right)-H\left(y_{j}, v\left(y_{j}\right)\right)\right] \\
U_{2} & =\sum_{j \geq 0} x_{0}^{-\mathcal{C}} x_{j}^{\mathcal{C}}\left[\widehat{E}\left(x_{0}\right) \widehat{E}\left(x_{j}\right)^{-1}-\widehat{E}\left(x_{0}\right) \widehat{E}\left(y_{j}\right)^{-1}\right] H\left(y_{j}, v\left(y_{j}\right)\right) \\
U_{3} & =\sum_{j \geq 0}^{-\mathcal{C}} x_{0}^{-\mathcal{C}} x_{j}^{\mathcal{C}}\left[I-y_{j}^{\mathcal{C}} x_{j}^{-\mathcal{C}}\right] \widehat{E}\left(x_{0}\right) \widehat{E}\left(y_{j}\right)^{-1} H\left(y_{j}, v\left(y_{j}\right)\right) .
\end{aligned}
$$

To bound $U_{1}$, we write first

$$
H\left(x_{j}, u\left(x_{j}\right)\right)-H\left(y_{j}, v\left(y_{j}\right)\right)=H\left(x_{j}, u\left(x_{j}\right)\right)-H\left(y_{j}, u\left(x_{j}\right)\right)+H\left(y_{j}, u\left(x_{j}\right)\right)-H\left(y_{j}, v\left(y_{j}\right)\right) .
$$

Using (9) and taking into account that $u \in \mathcal{H}_{\delta}$, that $m \geq p+2$ and that $x_{j} \sim y_{j}$ when $j \rightarrow \infty$ (see Proposition [3.7), we obtain that there are constants $c_{1}, c_{2}>0$ (independent of $\delta, x_{0}, u, v$ ) such that

$$
\begin{aligned}
\left\|H\left(x_{j}, u\left(x_{j}\right)\right)-H\left(y_{j}, u\left(x_{j}\right)\right)\right\| & \leq c_{1}\left|x_{j}\right|^{k+p+m-1}\left|x_{j}-y_{j}\right|, \\
\left\|H\left(y_{j}, u\left(x_{j}\right)\right)-H\left(y_{j}, v\left(y_{j}\right)\right)\right\| & \leq c_{2}\left|x_{j}\right|^{k+p+1}\left\|v\left(y_{j}\right)-u\left(x_{j}\right)\right\| .
\end{aligned}
$$

On the other hand, using similar arguments, there exists $c_{3}>0$ such that

$$
\begin{aligned}
\left\|v\left(y_{j}\right)-u\left(x_{j}\right)\right\| & \leq\left\|v\left(y_{j}\right)-v\left(x_{j}\right)\right\|+\left\|v\left(x_{j}\right)-u\left(x_{j}\right)\right\| \\
& \leq c_{3}\left(\left|x_{j}\right|^{m-p-2}\left|y_{j}-x_{j}\right|+\left|x_{j}\right|^{m-1} \mathbf{n}(v-u)\right) .
\end{aligned}
$$

Thus, by Lemma 3.8 and equation (11), and using the fact that $m \geq p+2-\sigma$, we get finally that there exists $B_{1}>0$, such that, if $\delta>0$ is small enough and $x_{0} \in S_{\delta}$ 
and $u, v \in \mathcal{H}_{\delta}$ then

$$
\left\|U_{1}\right\| \leq B_{1}\left|x_{0}\right|^{m} \mathbf{n}(v-u) .
$$

To bound $U_{2}$, put $\widehat{E}(x)=\exp (R(x))$ with

$$
R(x)=-\int \frac{\mathcal{D}(x)}{x^{p+1}} d x=\operatorname{diag}\left(r_{2}(x), \ldots, r_{n}(x)\right),
$$

and $r_{l}(x)=\frac{d_{l 0}}{\left(p-\nu_{l}\right) x^{p-\nu_{l}}} \tilde{r}_{l}(x)$, where $\tilde{r}_{l}(x) \in \mathbb{C}[x]$ is a polynomial of degree at most $p-\nu_{l}-1$ such that $\tilde{r}_{l}(0)=1$, for $l=2, \ldots, n$. Observe that $d_{l 0} \neq 0$ if and only if $r_{l}(x) \not \equiv 0$. Since $x_{1}=x_{0}-x_{0}^{k+p+1}+O\left(x_{0}^{k+p+1} u\left(x_{0}\right), x_{0}^{k+2 p+2}\right)$, we have

$$
r_{l}\left(x_{1}\right)=r_{l}\left(x_{0}\right)+d_{l 0} x_{0}^{k+\nu_{l}}\left(1+\theta_{u}\left(x_{0}\right)\right), l=2, \ldots, n,
$$

where $\left\|\theta_{u}\left(x_{0}\right)\right\| \leq c_{4}\left|x_{0}\right|$ for some constant $c_{4}>0$ independent of $u$. Since $\operatorname{Re}\left(d_{l 0} x_{1}^{k+\nu_{l}}\right)>0$ if $r_{l}(x) \not \equiv 0$ (by the definition of the interval $I$ and by Proposition [3.7), we obtain, by induction on $j \geq 1$, that $\operatorname{Re}\left(r_{l}\left(x_{0}\right)-r_{l}\left(x_{j}\right)\right) \leq 0$ and $\operatorname{Re}\left(r_{l}\left(x_{0}\right)-r_{l}\left(y_{j}\right)\right) \leq 0$ for $\left|x_{0}\right| \leq \delta$ sufficiently small and for all $2 \leq l \leq n$ and all $j \geq 1$. Therefore, if we put

$$
\widehat{E}\left(x_{0}\right) \widehat{E}\left(x_{j}\right)^{-1}-\widehat{E}\left(x_{0}\right) \widehat{E}\left(y_{j}\right)^{-1}=\exp (a)-\exp (b),
$$

where $a=R\left(x_{0}\right)-R\left(x_{j}\right), b=R\left(x_{0}\right)-R\left(y_{j}\right)$, then all diagonal entries of $a$ and $b$ are in the half space $\{z: \operatorname{Re}(z) \leq 0\}$ and thus

$$
\left\|\widehat{E}\left(x_{0}\right) \widehat{E}\left(x_{j}\right)^{-1}-\widehat{E}\left(x_{0}\right) \widehat{E}\left(y_{j}\right)^{-1}\right\| \leq|a-b| \max _{\xi \in[a, b]}\|\exp \xi\| \leq\left\|R\left(y_{j}\right)-R\left(x_{j}\right)\right\| .
$$

Finally, since $R^{\prime}(x)=O\left(|x|^{-p-1}\right)$ for $x \in S_{\delta}$, we conclude, using $x_{j} \sim y_{j}$, that there exists a constant $c_{5}>0$ independent of $\delta, x_{0}, u, v$ such that

$$
\left\|\widehat{E}\left(x_{0}\right) \widehat{E}\left(x_{j}\right)^{-1}-\widehat{E}\left(x_{0}\right) \widehat{E}\left(y_{j}\right)^{-1}\right\| \leq c_{5} \frac{\left|y_{j}-x_{j}\right|}{\left|x_{j}\right|^{p+1}}
$$

for all $j$. Then, applying Lemma 3.8 and equations (9) and (11), there exists $B_{2}>0$ such that, if $\delta>0$ is small enough and $x_{0} \in S_{\delta}$ and $u, v \in \mathcal{H}_{\delta}$ then

$$
\left\|U_{2}\right\| \leq B_{2}\left|x_{0}\right|^{m} \mathbf{n}(u-v) \text {. }
$$

To bound $U_{3}$, we use

$$
\left\|I-y_{j}^{\mathcal{C}} x_{j}^{-\mathcal{C}}\right\|=\left\|I-\exp \left(\mathcal{C} \log \frac{y_{j}}{x_{j}}\right)\right\| \leq c_{6} \frac{\left|y_{j}-x_{j}\right|}{\left|x_{j}\right|},
$$

with some constant $c_{6}>0$. Again by Lemma 3.8, equations (9) and (11) and the fact than $x_{j} \sim y_{j}$, there exists $B_{3}>0$ such that, if $\delta>0$ is small enough and $x_{0} \in S_{\delta}$ and $u, v \in \mathcal{H}_{\delta}$ then

$$
\left\|U_{3}\right\| \leq B_{3}\left|x_{0}\right|^{m} \mathbf{n}(u-v) .
$$

Summarizing, we have for any $u, v \in \mathcal{H}_{\delta}$ and every $x \in S_{\delta}$,

$$
\|T u(x)-T v(x)\| \leq\left(B_{1}+B_{2}+B_{3}\right)|x|^{m} \mathbf{n}(u-v) .
$$

We conclude that $T: \mathcal{H}_{\delta} \rightarrow \mathcal{H}_{\delta}$ is a contraction map if $\delta$ is small enough.

Finally, notice that we can rewrite the map $T$ as

$$
\begin{aligned}
T u\left(x_{0}\right) & =E\left(x_{0}\right) \sum_{j \geq 0}\left(E\left(x_{j}\right)^{-1} u\left(x_{j}\right)-E\left(x_{j+1}\right)^{-1} \bar{F}\left(x_{j}, u\left(x_{j}\right)\right)\right) \\
& =u\left(x_{0}\right)-E\left(x_{0}\right) E\left(x_{1}\right)^{-1} \bar{F}\left(x_{0}, u\left(x_{0}\right)\right)+E\left(x_{0}\right) E\left(x_{1}\right)^{-1} T u\left(x_{1}\right) .
\end{aligned}
$$


The first equality shows that if $u \in \mathcal{H}_{\delta}$ satisfies (7) then $u$ is a fixed point of $T$. The second one shows the converse.

End of the proof of Proposition 3.5. Fix $m \geq m_{0}$ and $\eta \in I$. Applying Proposition [3.9. there exists some $\delta=\delta(m, \eta)>0$ and a unique $u_{m, \delta^{\prime}} \in \mathcal{H}_{\eta, \delta^{\prime}}^{m}$ for any $0<\delta^{\prime} \leq \delta$ which is a solution of the invariance equation (77). Moreover, by Proposition 3.7 the orbit of any point by the map $f_{u_{m, \delta^{\prime}}}$ converges to 0 . This shows statement (a) of Proposition 3.5. Statement (b) follows once we remark that, if $\delta^{\prime}<\delta$ and $u \in \mathcal{H}_{\eta, \delta}^{m}$ satisfies equation (77), then the restriction of $u$ to $S\left(\tau, \eta, \delta^{\prime}\right)$ belongs to $\mathcal{H}_{\eta, \delta^{\prime}}^{m}$ and satisfies also equation (7) by Proposition 3.7 Let us prove finally statement (c). By Proposition 3.7. if we put $\tilde{v}=\left.v\right|_{S\left(\tau, \eta, \delta^{\prime}\right)}$ then $f_{\tilde{v}}\left(S\left(\tau, \eta, \delta^{\prime}\right)\right) \subset S\left(\tau, \eta, \delta^{\prime}\right)$ and, since $\psi(x)=(x, v(x))$ is a parabolic curve of $F, \tilde{v}$ satisfies equation (77). By Proposition 3.9 we have then $\tilde{v}=u_{m, \delta^{\prime}}$.

\section{TWO-DIMENSIONAL CASE}

Consider $F \in \operatorname{Diff}_{1}\left(\mathbb{C}^{2}, 0\right)$ and let $\Gamma$ be a formal invariant curve of $F$ which is not contained in the set of fixed points of $F$. In this paragraph we show how to use the results in the preceding paragraph in order to prove Theorem 11. The proof consists in two steps. In the first one, we reduce the general situation to the case where $(F, \Gamma)$ is in Ramis-Sibuya form. This reduction, which can already be found in 1, 6] and that we briefly report below, will also be obtained as a particular case of a general result in any dimension which we discuss in section 6 (see Theorem 6.1). In the second step, we prove that if $(F, \Gamma)$ is in RS-form then Theorem 3.3 applies either for $F$ or for $F^{-1}$ and we find a parabolic curve of $F$ (attracting or repelling) asymptotic to $\Gamma$.

The reduction to RS-form is not much more than the well known result of reduction of singularities of the formal vector field $X=\log F$ along $\Gamma$ (see [27] or the book [10] for a more recent presentation): after a finite composition $\Phi:\left(\mathbb{C}^{2}, 0\right) \rightarrow\left(\mathbb{C}^{2}, 0\right)$ of local blow-ups centered at the infinitely near points of $\Gamma$, the transform of $X$ is written as $\Phi^{*} X=x^{k} \bar{X}$, where $\{x=0\}$ is the exceptional divisor of $\Phi, \bar{X}(0)=0$ and the linear part $D_{0} \bar{X}$ is non-nilpotent; moreover, the strict transform $\widetilde{\Gamma}$ of $\Gamma$ by $\Phi$ is non-singular, transversal to $\{x=0\}$ and invariant for $\Phi^{*} X$. Since $\nu_{0}\left(\Phi^{*} X\right) \geq \nu_{0}(X)$ and the operation of taking the infinitesimal generator is compatible with blow-ups at singular points (see [6]), $\Phi^{*} X$ is the infinitesimal generator of the transformed diffeomorphism $\widetilde{F} \in \operatorname{Diff}_{1}\left(\mathbb{C}^{2}, 0\right)$ satisfying $\Phi \circ \widetilde{F}=F \circ \Phi$. From the fact that $D_{0} \bar{X}$ is non-nilpotent, and up to several additional blow-ups, we deduce that $\widetilde{F}$ is in RS-form. Finally, if $\widetilde{\varphi}: \Delta \rightarrow \mathbb{C}^{2}$ is a parabolic curve of $\widetilde{F}$ asymptotic to $\widetilde{\Gamma}$ then $\varphi=\Phi \circ \widetilde{\varphi}$ is a parabolic curve of $F$ asymptotic to $\Gamma$.

Concerning the second step, we can, in fact, give a better description of how many parabolic curves a diffeomorphism in RS-form may have:

Proposition 4.1. Assume that $(F, \Gamma)$ is in $R S$-form and let $k+1$ be the order of $F$ and $p$ the Poincaré rank of $(F, \Gamma)$. Then we have:

(a) If $p=0$ then there are $k$ attracting and $k$ repelling parabolic curves of $F$ asymptotic to $\Gamma$.

(b) If $1 \leq p<k$ then there are $p$ attracting and $p$ repelling parabolic curves of $F$ asymptotic to $\Gamma$. 
(c) If $k<p$ there is at least one attracting and one repelling parabolic curve of $F$ asymptotic to $\Gamma$.

(d) If $1 \leq p=k$ then there are $p$ attracting or $p$ repelling parabolic curves of $F$ asymptotic to $\Gamma$.

Proof. Statement (a) corresponds to the Briot-Bouquet case and is already proved in [17. It is also an immediate consequence of Theorem 3.3 since in this case the saddle domain is the whole complex plane $\mathbb{C}$. Let us assume that $p \geq 1$. Choose RS-coordinates $(x, y)$ at $0 \in \mathbb{C}^{2}$ such that $F$ is written as

$$
F(x, y)=\left(x-x^{k+p+1}(1+o(1)), y+x^{k}\left(a y+O(x)+O\left(y^{2}\right)\right)\right)
$$

where $a \in \mathbb{C}^{*}$. These coordinates are also RS-coordinates for the inverse diffeomorphism $F^{-1}$, which is written as

$$
F^{-1}(x, y)=\left(x+x^{k+p+1}(1+o(1)), y+x^{k}\left(-a y+O(x)+O\left(y^{2}\right)\right)\right) .
$$

Thus, both $F$ and $F^{-1}$ have the same saddle domain $V=\left\{\operatorname{Re}\left(\frac{a}{x^{p}}\right)>0\right\}$ in these coordinates, whereas the attracting directions for $F$ (respectively for $F^{-1}$ ) are determined by the $(k+p)$-th roots of 1 (respectively of -1$)$. Notice also that we only need to prove the statement for attracting parabolic curves in the cases (b) and (c). Write $V=V_{1} \cup \cdots \cup V_{p}$ as a union of disjoint open sectors of opening $\pi / p$. If $p<k$, since the angle between two consecutive attracting directions is $2 \pi /(k+p)<\pi / p$, each $V_{j}$ contains at least one attracting direction. Theorem 3.3 thus implies (b). Let us show (c) by contradiction. Assume that $p>k$ and there is no attracting direction contained in $V$. Then, since $2 \pi /(p+k)>\pi / p$, the sector between any pair of consecutive attracting directions should contain one of the sectors $V_{j}$, showing that there are as much sectors $V_{j}$ as attracting directions, which is impossible. Finally, if $p=k$ the angle between two consecutive attracting directions is $\pi / p$. Then, either each $V_{j}$ contains exactly one such attracting direction or the attracting directions are in the boundary of the sectors $V_{j}$. But, in this last case, the bisectrices of the sectors $V_{j}$ are attracting directions of $F^{-1}$. This shows (d), using Theorem 3.3 .

\section{BLOW-UPS AND RAMIFICATIONS OF DIFFEOMORPHISMS}

A consequence of the property of a parabolic curve $\varphi$ being asymptotic to a formal curve $\Gamma$ is that these two objects share the same sequence of iterated tangents. This is a very natural notion (partially motivated by the corresponding concept for trajectories of real vector fields developed in [12, 13]) which means that, for any sequence of finitely many punctual blow-ups $\pi: \widetilde{\mathbb{C}}^{n} \rightarrow \mathbb{C}^{n}$, the transformed map

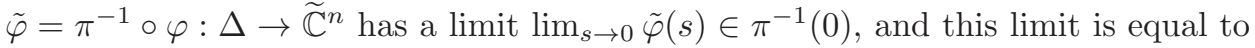
the corresponding point of the transformed of $\Gamma$ by $\pi$.

This property suggests that the operation of blowing-up is a good tool for the problem of existence of parabolic curves of $F \in \operatorname{Diff}_{1}\left(\mathbb{C}^{n}, 0\right)$ asymptotic to a formal invariant curve $\Gamma$. This approach works satisfactorily in dimension two, as we have shown in section 4 , since we have reduction of singularities for vector fields and punctual blow-ups are compatible with the operation of taking the infinitesimal generator of $F$.

In higher dimension, the situation is much less clear since we do not have reduction of singularities of vector fields by blowing-up points in the sequence of iterated tangents of an invariant formal curve; not even by blowing-up invariant centers of 
bigger dimension: there are examples of (polynomial) vector fields in $\mathbb{C}^{3}$ with a formal invariant curve $\Gamma$ at the origin whose strict transform by any sequence of blow-ups of points or invariant analytic non-singular curves at the corresponding point of the transform of $\Gamma$ has always a nilpotent linear part (see Example 1.2 in [22]). Together with blow-ups, we also need to consider ramifications.

Let $Z$ be a germ of a non-singular analytic submanifold of $\mathbb{C}^{n}$ at the origin. Let $U$ be an open neighborhood of $0 \in \mathbb{C}^{n}$ where $Z$ has a representative $Z^{\prime} \subset U$ which is a non-singular analytic submanifold of $U$. We will denote by $\pi_{Z}: M \rightarrow U$ the blow-up of $U$ with center $Z^{\prime}$, which we will simply call the blow-up with center $Z$. The fiber $\pi_{Z}^{-1}(0)$ can be identified with the projective space over the normal space, $T_{0} \mathbb{C}^{n} / T_{0} Z$, of $Z$ at the origin.

Let $X$ be a formal vector field at $0 \in \mathbb{C}^{n}$ with $\nu_{0}(X) \geq 1$. A germ $Z$ of nonsingular analytic submanifold is invariant for $X$ if $X(g) \in I(Z)$ for any $g \in I(Z)$, where $I(Z)$ denotes the ideal of holomorphic germs vanishing on $Z$. In this case, the multiplicity of $X$ along $Z$ is defined as

$$
\nu_{Z}(X)=\min \left\{l \geq 1: X(I(Z)) \subset I(Z)^{l} \backslash I(Z)^{l+1}\right\} .
$$

Suppose now that $\Gamma$ is a formal invariant curve of $X$ not contained in the singular locus of $X$. A germ of holomorphic map $\phi:\left(\mathbb{C}^{n}, 0\right) \rightarrow\left(\mathbb{C}^{n}, 0\right)$ will be called a permissible transformation for the pair $(X, \Gamma)$ if it is of one of the following types:

1. The germ of a holomorphic diffeomorphism.

2. Let $Z$ be a germ of non-singular analytic submanifold at $0 \in \mathbb{C}^{n}$, transversal to $\Gamma$ at 0 , invariant for $X$ and such that it satisfies $\nu_{Z}(X) \geq \nu_{0}(X)$. Let $\pi_{Z}: M \rightarrow U$ be the blow-up with center $Z$ and let $p \in \pi_{Z}^{-1}(0)$ be the point corresponding to the tangent of $\Gamma$. Then there is an analytic chart $\tau$ of $M$ at $p$ so that $\phi$ is the germ of $\pi_{Z} \tau^{-1}$ at $0 \in \mathbb{C}^{n}$. We will say that $Z$ is a permissible center and that $\phi$ is a permissible blow-up.

3. The curve $\Gamma$ is non-singular, there are analytic coordinates $\mathbf{z}=\left(z_{1}, \ldots, z_{n}\right)$ at $0 \in \mathbb{C}^{n}$ such that $Z=\left\{z_{1}=0\right\}$ is invariant for $X$ and transversal to $\Gamma$ and $\phi$ is the map $\phi(\mathbf{z})=\left(z_{1}^{q}, z_{2}, \ldots, z_{n}\right)$ for some $q \in \mathbb{N}_{>0}$. We will say that $\phi$ is a permissible q-ramification (with respect to $Z$ ).

In the last two cases, the non-singular hypersurface $E_{\phi}=\phi^{-1}(Z)$ is called the exceptional divisor of $\phi$. For convenience, $E_{\phi}=\emptyset$ in the case where $\phi$ is a diffeomorphism. Notice that a permissible transformation $\phi$ is a local diffeomorphism at every point in the complement of $E_{\phi}$.

Observe that the notion of permissible center depends essentially on $X$ (except for the transversality with $\Gamma$ ); however, the notion of permissible blow-up depends on both $X$ and $\Gamma$, since it chooses the point in the divisor corresponding to the tangent of $\Gamma$. Note that $Z=\{0\}$ is a permissible center for $(X, \Gamma)$ as long as 0 is a singular point of $X$.

Proposition 5.1. Let $\phi:\left(\mathbb{C}^{n}, 0\right) \rightarrow\left(\mathbb{C}^{n}, 0\right)$ be a permissible transformation for $(X, \Gamma)$. There exist a unique formal curve $\widetilde{\Gamma}$ at $0 \in \mathbb{C}^{n}$ such that $\phi^{*} \Gamma \subset \widetilde{\Gamma}$ (where $\phi^{*} \Gamma=\{g \circ \phi: g \in \Gamma\}$ ) and a unique formal vector field $\widetilde{X}$ at $0 \in \mathbb{C}^{n}$ such that $\phi_{*} \widetilde{X}=X$, which also has $\widetilde{\Gamma}$ as an invariant curve and satisfies $\nu_{0}(\widetilde{X}) \geq \nu_{0}(X)$.

We will call $\widetilde{X}$ and $\widetilde{\Gamma}$ the transforms of $X$ and $\Gamma$ by $\phi$, respectively.

Proof. The case where $\phi$ is a germ of a diffeomorphism is clear. 
Suppose that $\phi$ is a permissible blow-up with center $Z$. Take analytic coordinates $\mathbf{z}=\left(z_{1}, z_{2}, \ldots, z_{n}\right)$ so that the tangent of $\Gamma$ corresponds to the $z_{1}$-axis and so that $Z=\left\{z_{1}=z_{2}=\cdots=z_{t}=0\right\}$ where $t=\operatorname{codim} Z$ (thus $I(Z)$ is generated by $\left.z_{1}, \ldots, z_{t}\right)$. Then we may write $\phi:\left(\mathbb{C}^{n}, 0\right) \rightarrow\left(\mathbb{C}^{n}, 0\right)$ as

$$
\phi(\mathbf{z})=\left(z_{1}, z_{1} z_{2}, \ldots, z_{1} z_{t}, z_{t+1}, \ldots, z_{n}\right) .
$$

Let $\gamma(s)=\left(\gamma_{1}(s), \ldots, \gamma_{n}(s)\right) \in \mathbb{C}[[s]]^{n}$ be a parametrization of $\Gamma$ in the coordinates z. Then

$$
m=\nu_{0}(\Gamma)=\nu\left(\gamma_{1}(s)\right)<\nu\left(\gamma_{j}(s)\right) \text { for } j=2, \ldots, n,
$$

where $\nu$ denotes the order in $s$. Also,

$$
\widetilde{\gamma}(s)=\left(\gamma_{1}(s), \frac{\gamma_{2}(s)}{\gamma_{1}(s)}, \ldots, \frac{\gamma_{t}(s)}{\gamma_{1}(s)}, \gamma_{t+1}(s), \ldots, \gamma_{n}(s)\right) \in \mathbb{C}[[s]]^{n}
$$

is a parametrization of the formal curve $\widetilde{\Gamma}$ which satisfies $\phi^{*} \Gamma \subset \widetilde{\Gamma}$. The uniqueness of $\widetilde{\Gamma}$ can be seen as follows: if $\bar{\gamma}(s)=\left(\bar{\gamma}_{1}(s), \bar{\gamma}_{2}(s), \ldots, \bar{\gamma}_{n}(s)\right)$ is a parametrization of another formal curve $\bar{\Gamma}$ satisfying $\phi^{*} \Gamma \subset \bar{\Gamma}$ then we will have that $\phi \circ \bar{\gamma}(s)$ is another parametrization of $\Gamma$ and necessarily $\phi \circ \bar{\gamma}(s)=\gamma(\sigma(s))$ where $\sigma(s) \in \mathbb{C}[[s]]$. Using the expression of $\phi$ and equation (12) one shows that also $\bar{\gamma}(s)=\widetilde{\gamma}(\sigma(s))$ and we are done.

Write $X=\sum_{i=1}^{n} a_{i}(\mathbf{z}) \frac{\partial}{\partial z_{i}}$. Since $\Gamma$ is invariant and not contained in the singular locus of $X,\left.X\right|_{\gamma(s)} \neq 0$ is a multiple of $\gamma^{\prime}(s)$ (see section 2) and hence

$$
\nu\left(a_{1}(\gamma(s))\right)<\nu\left(a_{j}(\gamma(s))\right) \text { for } j=2, \ldots, n .
$$

On the other hand, the condition of $Z$ being invariant implies that, for $j=1, \ldots, t$, $a_{j}(\mathbf{z}) \in I(Z)$ and hence $a_{j}(\phi(\mathbf{z}))$ is divisible by $z_{1}$. Using this property, the vector field $\tilde{X}=\sum_{i=1}^{n} \tilde{a}_{i}(\mathbf{z}) \frac{\partial}{\partial z_{i}}$ defined by

$$
\begin{cases}\tilde{a}_{j}(\mathbf{z})=\frac{a_{j}(\phi(\mathbf{z}))-z_{j} a_{1}(\phi(\mathbf{z}))}{z_{1}}, & \text { for } j=2, \ldots, t ; \\ \tilde{a}_{j}(\mathbf{z})=a_{j}(\phi(\mathbf{z})), & \text { for } j \in\{1, t+1, \ldots, n\},\end{cases}
$$

is formal and satisfies $\phi_{*} \tilde{X}=X$. Let us show that $\nu_{0}(\tilde{X}) \geq \nu_{0}(X)$. Denote by $r=\nu_{0}(X)$. Since $Z$ is a permissible center, we have $r \leq \nu_{Z}(X)$ and thus $X\left(z_{j}\right)=$ $a_{j}(\mathbf{z}) \in I(Z)^{r}$ for $j=1, \ldots, t$. Then, according to the expression of the transformed vector field $\widetilde{X}$, it suffices to show that for $j \in\{2, \ldots, t\}$ the component $a_{j}(\mathbf{z})$ does not contain a monomial of the form $c z_{1}^{r}$ with $c \neq 0$. Suppose, on the contrary, that such a monomial appears in $a_{j}(\mathbf{z})$ for some $j \in\{2, \ldots, t\}$. Then we will have that the series $a_{j}(\gamma(s)) \in \mathbb{C}[[s]]$ has order $r m$. But since $\nu\left(a_{l}(\gamma(s))\right) \geq \nu\left(a_{l}\right) \nu_{0}(\Gamma) \geq r m$ for any $l \in\{1, \ldots, n\}$, this is a contradiction with (13).

Assume now that $\phi$ is a permissible $q$-ramification, written in some coordinates $\mathbf{z}$ as $\phi(\mathbf{z})=\left(z_{1}^{q}, z_{2}, \ldots, z_{n}\right)$. Consider a parametrization of $\Gamma$ of the form $\gamma(s)=\left(s, \gamma_{2}(s), \ldots, \gamma_{n}(s)\right)$ in these coordinates (recall that, from the definition of permissible ramification, $\Gamma$ is non-singular). Then

$$
\widetilde{\gamma}(s)=\left(s, \gamma_{2}\left(s^{q}\right), \ldots, \gamma_{n}\left(s^{q}\right)\right) \in \mathbb{C}[[s]]^{n}
$$

is a parametrization of a formal curve $\widetilde{\Gamma}$ satisfying $\phi^{*} \Gamma \subset \widetilde{\Gamma}$. Uniqueness of $\widetilde{\Gamma}$ comes from the property of $\Gamma$ being non-singular: $\Gamma$ is generated by the series $z_{j}-\gamma_{j}\left(z_{1}\right)$ for $j=2, \ldots, n$ and thus, if $\phi^{*} \Gamma \subset \widetilde{\Gamma}$, then $\widetilde{\Gamma}$ must be the (prime) ideal generated by $z_{j}-\gamma_{j}\left(z_{1}^{q}\right)$ for $j=2, \ldots, n$. 
On the other hand, being $Z=\left\{z_{1}=0\right\}$ invariant for $X$, if we write $X=$ $\sum_{i=1}^{n} a_{i}(\mathbf{z}) \frac{\partial}{\partial z_{i}}$ then we have $a_{1}(\mathbf{z})=z_{1} \bar{a}_{1}(\mathbf{z})$, where $\bar{a}_{1}(\mathbf{z})$ is a formal series. We can check that the formal vector field $\tilde{X}$ defined by

$$
\widetilde{X}=\frac{z_{1} \bar{a}_{1}(\phi(\mathbf{z}))}{q} \frac{\partial}{\partial z_{1}}+\sum_{i=2}^{n} a_{i}(\phi(\mathbf{z})) \frac{\partial}{\partial z_{i}}
$$

satisfies $\phi_{*} \tilde{X}=X$ and, clearly, $\nu_{0}(\tilde{X}) \geq \nu_{0}(X)$.

It is worth to notice that Proposition 5.1 remains true, except for the uniqueness of the curve $\widetilde{\Gamma}$ satisfying $\phi^{*} \Gamma \subset \widetilde{\Gamma}$, if the condition of $\Gamma$ being non-singular in the definition of permissible ramification is removed (consider, for example, $\Gamma=$ $\left(y^{2}-x^{3}\right)$ and $\left.\phi(x, y)=\left(x^{2}, y\right)\right)$.

Remark 5.2. Observe that finite truncations of the expression of the transform of a vector field by a permissible transformation are finitely determined: if $\phi$ is a permissible transformation for $(X, \Gamma)$ and $N \in \mathbb{N}$, there exists $N^{\prime} \in \mathbb{N}$ such that if $\phi$ is also "permissible" for another formal vector field $Y$ (considering just the conditions in the definition of permissible center concerning the vector field and not the formal invariant curve) and $J_{N^{\prime}} Y=J_{N^{\prime}} X$ then for the transforms $\widetilde{X}, \widetilde{Y}$ of $X, Y$ we have $J_{N} \widetilde{Y}=J_{N} \widetilde{X}$.

Consider now a diffeomorphism $F \in \operatorname{Diff}_{1}\left(\mathbb{C}^{n}, 0\right)$ with a formal invariant curve $\Gamma$ not contained in the set of fixed points of $F$. A germ of holomorphic map $\phi:\left(\mathbb{C}^{n}, 0\right) \rightarrow\left(\mathbb{C}^{n}, 0\right)$ will be called a permissible transformation for $(F, \Gamma)$ if it is a permissible transformation for $(\log F, \Gamma)$. In the following proposition, we show that permissible transformations are compatible with the operation of taking the infinitesimal generator of $F$ and with the existence of parabolic curves.

Proposition 5.3. Let $\phi:\left(\mathbb{C}^{n}, 0\right) \rightarrow\left(\mathbb{C}^{n}, 0\right)$ be a permissible transformation for $(F, \Gamma)$, and let $\widetilde{X}$ and $\widetilde{\Gamma}$ be the transforms of $\log F$ and $\Gamma$ by $\phi$. There exists a unique diffeomorphism $\widetilde{F} \in \operatorname{Diff}_{1}\left(\mathbb{C}^{n}, 0\right)$ such that $\phi \circ \widetilde{F}=F \circ \phi$, which also satisfies $\log \widetilde{F}=\widetilde{X}$. Moreover, if $\widetilde{\varphi}: \Delta \rightarrow \mathbb{C}^{n}$ is a parabolic curve of $\widetilde{F}$ asymptotic to $\widetilde{\Gamma}$ such that $\widetilde{\varphi}(\Delta)$ is contained in the domain of $\phi$ and does not intersect $E_{\phi}$, then $\varphi=\phi \circ \widetilde{\varphi}$ is a parabolic curve of $F$ asymptotic to $\Gamma$ (provided $\varphi$ is injective).

The pair $(\widetilde{F}, \widetilde{\Gamma})$ is called the transform of $(F, \Gamma)$ by $\phi$.

Proof. If $\phi$ is a germ of a diffeomorphism, the result is clear.

Assume that $\phi$ is a permissible blow-up with center $Z$. Consider analytic coordinates $\mathbf{z}=\left(z_{1}, z_{2}, \ldots, z_{n}\right)$ so that $\Gamma$ is tangent to the $z_{1}$-axis, $Z=\left\{z_{1}=z_{2}=\right.$ $\left.\cdots=z_{t}=0\right\}$ and so that $\phi(\mathbf{z})=\left(z_{1}, z_{1} z_{2}, \ldots, z_{1} z_{t}, z_{t+1}, \ldots, z_{n}\right)$. The condition $\phi \circ \widetilde{F}=F \circ \phi$ can be written as

$$
z_{j} \circ \widetilde{F}(\mathbf{z})= \begin{cases}\frac{z_{j} \circ F(\phi(\mathbf{z}))}{z_{1} \circ F(\phi(\mathbf{z}))}, & \text { if } j=2, \ldots, t \\ z_{j} \circ F(\phi(\mathbf{z})), & \text { if } j \in\{1, t+1, \ldots, n\} .\end{cases}
$$

As in the case of formal curves, the invariance of $Z$ for $\log F$ is equivalent to the condition $g \circ F \in I(Z)$ for any $g \in I(Z)$ and therefore $z_{j} \circ F(\phi(\mathbf{z}))$ is divisible by $z_{1}$ for $j=1, \ldots, t$. Moreover, $z_{1} \circ F(\phi(\mathbf{z}))=z_{1}(1+A(\mathbf{z}))$ with $A(0)=0$ and we conclude that $\widetilde{F} \in \operatorname{Diff}_{1}\left(\mathbb{C}^{n}, 0\right)$. In order to prove that $\log \widetilde{F}=\widetilde{X}$, observe that 
$\nu_{0}(\widetilde{X}) \geq \nu_{0}(X) \geq 2$ by Proposition [5.1, where $X=\log F$. Then $\operatorname{Exp} \widetilde{X}$ is a formal tangent to identity diffeomorphism at $0 \in \mathbb{C}^{n}$. Moreover, since $\widetilde{X}^{j}(g \circ \phi)=X^{j}(g) \circ \phi$ for any $g \in \widehat{\mathcal{O}}_{n}$ and any $j \geq 1$, using (2) we have that $\operatorname{Exp} \widetilde{X}$ satisfies formally $\phi \circ \operatorname{Exp} \widetilde{X}=F \circ \phi$ and hence $\operatorname{Exp} \widetilde{X}=\widetilde{F}$.

To finish the proof for permissible blow-ups, let $\widetilde{\varphi}: \Delta \rightarrow \mathbb{C}^{n}$ be a parabolic curve of $\widetilde{F}$ whose image does not intersect $E_{\phi}$. Since $\phi \circ \widetilde{F}=F \circ \phi$, we deduce easily that $\varphi=\phi \circ \widetilde{\varphi}$ is a a parabolic curve of $F$ (notice that in this case $\phi$ is injective outside $\left.E_{\phi}\right)$. On the other hand, if $\widetilde{\varphi}$ is asymptotic to $\widetilde{\Gamma}$, i.e., $\mathbf{z} \circ \widetilde{\varphi}$ has asymptotic expansion at $s=0$ equal to $\widetilde{\gamma}(s) \in \mathbb{C}[[s]]^{n}$, where $\widetilde{\gamma}(s)$ is a parametrization of $\widetilde{\Gamma}$ in the given coordinates, then, being $\gamma(s)=\phi \circ \widetilde{\gamma}(s)$ a parametrization of $\Gamma, \varphi$ is asymptotic to $\Gamma$.

Assume now that $\phi$ is a permissible $q$-ramification, written in some coordinates $\mathbf{z}$ as $\phi(\mathbf{z})=\left(z_{1}^{q}, z_{2}, \ldots, z_{n}\right)$. If $\phi \circ \widetilde{F}=F \circ \phi$, using the expression of $\phi$ we obtain

$$
\left(z_{1} \circ \widetilde{F}(\mathbf{z})\right)^{q}=z_{1} \circ F(\phi(\mathbf{z})), \quad z_{j} \circ \widetilde{F}(\mathbf{z})=z_{j} \circ F(\phi(\mathbf{z})), j=2, \ldots, n,
$$

and since $z_{1} \circ F(\mathbf{z})=z_{1}(1+A(\mathbf{z}))$ with $A(0)=0$, by invariance of $\left\{z_{1}=0\right\}$, we conclude that $\widetilde{F} \in \operatorname{Diff}_{1}\left(\mathbb{C}^{n}, 0\right)$. The claim concerning the infinitesimal generator and the one concerning parabolic curves are proved as in the case of permissible blow-ups.

Remark 5.4. Observe that if $\phi$ is a permissible ramification and $\widetilde{\varphi}: \Delta \rightarrow \mathbb{C}^{n}$ is a parabolic curve, there is no need for the composition $\varphi=\phi \circ \widetilde{\varphi}$ to be injective; that is the reason of the hypothesis "provided $\varphi$ is injective" stated in the proposition.

\section{Reduction to RAmis-Sibuya Form}

In this section we show that for any $F \in \operatorname{Diff}_{1}\left(\mathbb{C}^{n}, 0\right)$ and any formal invariant curve $\Gamma$ of $F$, the pair $(F, \Gamma)$ can be reduced to Ramis-Sibuya form by permissible transformations.

Let $X$ be a formal vector field and $\Gamma$ be a formal invariant curve of $X$. A sequence of permissible transformations for $(X, \Gamma)$ is a composition

$$
\Phi=\phi_{l} \circ \phi_{l-1} \circ \cdots \circ \phi_{1}:\left(\mathbb{C}^{n}, 0\right) \rightarrow\left(\mathbb{C}^{n}, 0\right)
$$

such that $\phi_{1}$ is a permissible transformation for $(X, \Gamma)$ and, for $j=1, \ldots, l-1$, $\left(X_{j}, \Gamma_{j}\right)$ is the transform of $\left(X_{j-1}, \Gamma_{j-1}\right)$ by $\phi_{j}$ and $\phi_{j+1}$ is a permissible transformation for $\left(X_{j}, \Gamma_{j}\right)$. The last pair $\left(X_{l}, \Gamma_{l}\right)$ will be called the transform of $(X, \Gamma)$ by the sequence $\Phi$. We also define the total divisor of $\Phi$ as the set $E_{\Phi}=$ $\left(\phi_{l} \circ \phi_{l-1} \circ \cdots \circ \phi_{2}\right)^{-1}\left(E_{\phi_{1}}\right)$, which is a normal crossing divisor at $0 \in \mathbb{C}^{n}$. If $F \in \operatorname{Diff}_{1}\left(\mathbb{C}^{n}, 0\right)$ and $\Gamma$ is a formal invariant curve, we define the same concept of sequence of permissible transformations for $(F, \Gamma)$ and the corresponding transform $(\widetilde{F}, \widetilde{\Gamma})$ using $X=\log F$.

Theorem 6.1 (Reduction of a diffeomorphism to Ramis-Sibuya form). Let $F \in$ $\operatorname{Diff}_{1}\left(\mathbb{C}^{n}, 0\right)$, let $\Gamma$ be a formal invariant curve of $F$ and assume that $\Gamma$ is not contained in the set of fixed points of $F$. There is sequence $\Phi$ of permissible transformations for $(F, \Gamma)$ so that the transform $(\widetilde{F}, \widetilde{\Gamma})$ of $(F, \Gamma)$ by $\Phi$ is in Ramis-Sibuya form and $\operatorname{Fix}(\widetilde{F})=E_{\Phi}$.

The corresponding statement for vector fields is the following one. 
Theorem 6.2 (Reduction of a vector field to Ramis-Sibuya form). Let $X$ be a formal vector field at $0 \in \mathbb{C}^{n}$ with $X(0)=0$ and let $\Gamma$ be an invariant formal curve of $X$ not contained in the singular locus of $X$. There exists a sequence $\Phi$ of permissible transformations for $(X, \Gamma)$ and there are analytic coordinates $(x, \mathbf{y})=\left(x, y_{2}, \ldots, y_{n}\right)$ at $0 \in \mathbb{C}^{n}$ so that the total divisor of $\Phi$ is $E_{\Phi}=\{x=0\}$ and

(i) The transform of $\Gamma$ by $\Phi$ is non singular and tangent to the $x$-axis.

(ii) The transform of $X$ by $\Phi$ is written as

$$
\Phi^{*} X=x^{k}\left[x^{p+1} u(x, \mathbf{y}) \frac{\partial}{\partial x}+\left(c(x)+\left(\mathcal{D}(x)+x^{p} \mathcal{C}+O\left(x^{p+1}\right)\right) \mathbf{y}+O\left(\|\mathbf{y}\|^{2}\right)\right) \frac{\partial}{\partial \mathbf{y}}\right]
$$

where $u \in \mathbb{C}[[x, \mathbf{y}]]$ with $u(0,0) \neq 0, c(x) \in \mathbb{C}[[x]]^{n-1}$ with $c(0)=0, k \geq 0$ and either $p=0$ and $\mathcal{C} \neq 0$ or $p \geq 1$ and $\mathcal{D}(x)$ a diagonal matrix of polynomials of degree at most $p-1$ commuting with $\mathcal{C}$ and $\mathcal{D}(0) \neq 0$.

Any such sequence $\Phi$ will be called a reduction of $(X, \Gamma)$ to RS-form.

Theorem 6.1 is a consequence of Theorem 6.2 taking $X=\log F$ and using Proposition 5.3. Notice that in this case the number $k$ in equation (14) is at least 1 since $\nu_{0}\left(\Phi^{*} X\right) \geq 2$ by Proposition 5.1

Theorem 6.2 is, in essence, quite well known in the theory of reduction of singularities of vector fields as well as in the theory of systems of meromorphic ODEs with irregular singularity. It contains a weak form of "local uniformization" of $X$ along $\Gamma$, i.e. reduction to non-nilpotent linear part (in the context of real vector fields in dimension three, see Cano et al. 11 for a local uniformization along non-oscillating trajectories and also Panazzolo 22 for a global reduction of singularities). The particular form of equation (14) requires more than just a non-nilpotent linear part of the vector field. In our case (reduction along a formal invariant curve), that particular form is obtained, once we associate to $X$ a system of $n-1$ meromorphic ODEs after some initial blow-ups, from classical results in the theory of ODEs: see Turrittin [28] (for systems of linear ODEs), Braaksma [5] (for the non-linear case) or Cano et al. [12, 13] (for related statements for real vector fields in dimension three). However, we could not find an statement with the precise terms of Theorem 6.2 needed for the purposes of this article. Hence, for the sake of completeness, we provide here a self-contained proof to which we devote the rest of this section.

Let us first describe the situation after a punctual blow-up. Let $(x, \mathbf{y})$ be adapted coordinates for $\Gamma$ and let $\phi:\left(\mathbb{C}^{n}, 0\right) \rightarrow\left(\mathbb{C}^{n}, 0\right)$ be a permissible blow-up for $(X, \Gamma)$ at the origin. There is a constant vector $\xi \in \mathbb{C}^{n-1}$ so that $\phi$ is written, for some coordinates $(x, \widetilde{\mathbf{y}})$, as

$$
(x, \mathbf{y})=\phi(x, \widetilde{\mathbf{y}})=(x, x(\widetilde{\mathbf{y}}-\xi)) .
$$

The total transform of $X$ by $\phi$ can be written as $\widetilde{X}=x^{\nu_{0}(X)-1} \bar{X}$ where $x$ is a coordinate such that $E_{\phi}=\{x=0\}$ and $\bar{X}$ is a formal vector field singular at 0 (in fact $\nu_{0}(\tilde{X}) \geq \nu_{0}(X)$, see Proposition 5.1). We deduce that $\widetilde{X}$ is again singular at the origin, which is then a permissible center for the transform $(\widetilde{X}, \widetilde{\Gamma})$ of $(X, \Gamma)$. Performing in this way the blow-ups at the infinitely near points of $\Gamma$ and using resolution of singularities of curves, we can assume that $\Gamma$ is non-singular and that there is a system of adapted coordinates $(x, \mathbf{y})$ for $\Gamma$ such that $X=x^{e} \bar{X}$, where $\bar{X}$ is not divisible by $x$ and $e \geq 1$ if $\nu_{0}(X) \geq 2$ (and thus $\{x=0\}$ is invariant for $X$ ). 
Let $\gamma(x)=(x, \bar{\gamma}(x)) \in \mathbb{C}[[x]]^{n}$ be a parametrization of $\Gamma$ in these coordinates and write

$$
\bar{X}=a(x, \mathbf{y}) \frac{\partial}{\partial x}+\mathbf{b}(x, \mathbf{y}) \frac{\partial}{\partial \mathbf{y}},
$$

where $a(x, \mathbf{y}) \in \mathbb{C}[[x, \mathbf{y}]]$. By invariance of $\Gamma,\left.X\right|_{\gamma(x)}$ is collinear to the vector $\left(1, \bar{\gamma}^{\prime}(x)\right)$ and then $a(\gamma(x)) \neq 0$, since $\Gamma$ is not contained in the singular locus of $X$.

We may suppose that $\bar{X}$ is singular at the origin. In fact, if $\bar{X}(0) \neq 0$ we must have $e \geq 1$ and, since in this case $\Gamma$ is the unique formal solution of $\bar{X}$ at 0 and it is transversal to $\{x=0\}$, we must have $a(0)=a_{0} \neq 0$. After a new blow-up at the origin, and taking coordinates as in (15), the transform of $X$ is written as

$$
\widetilde{X}=x^{e-1}\left[x\left(a_{0}+O(x)\right) \frac{\partial}{\partial x}+\left(-a_{0} I_{n-1} \widetilde{\mathbf{y}}+O(x)\right) \frac{\partial}{\partial \widetilde{\mathbf{y}}}\right]
$$

which is of the required form (14) with $k=e-1 \geq 0$ and $p=0$.

Assume then that $\bar{X}$ is singular. Let $r$ be the multiplicity of the restriction $\left.\bar{X}\right|_{\Gamma}$ (notice that $r$ is the order of the series $a(\gamma(x))$ and hence $1 \leq r<\infty$ ). Let $\phi$ be the permissible blow-up at the origin written in coordinates as in (15), where in this case $\xi=\bar{\gamma}^{\prime}(0)$. The effect of this transformation on the vector field is that the exponent of $x$ increases in any monomial of $a(x, \mathbf{y})$ with positive degree in the $\mathbf{y}$-variables and in any monomial of the components of $\mathbf{b}(x, \mathbf{y})$ with degree at least two in the $\mathbf{y}$-variables. Thus, after several such blow-ups of points, the new $a$-component of the transform of $\bar{X}$ is of the form $x^{r} u(x, \mathbf{y})$ where $u(0,0) \neq 0$ and each monomial in the new $\mathbf{b}$-components of degree at least two in the $\mathbf{y}$-variables is divisible by $x^{r}$. Moreover, the monomials of degree 0 in the $\mathbf{y}$-variables of the new b-components can be assumed to be divisible by $x^{r}$ by means of a translation of the form $\widetilde{\mathbf{y}}=\mathbf{y}+Q(x)$, where $Q(x)$ is a polynomial truncation of $\bar{\gamma}(x) \in \mathbb{C}[[x]]^{n-1}$.

We conclude that, up to permissible blow-ups of points and changes of coordinates, the vector field $X$ may be written as

$$
X=x^{l}\left[x^{q+1} u(x, \mathbf{y}) \frac{\partial}{\partial x}+(c(x)+A(x) \mathbf{y}+\Theta(x, \mathbf{y})) \frac{\partial}{\partial \mathbf{y}}\right]
$$

where $l \geq 0, q \geq-1, u(0,0) \neq 0, c(0)=0$ and $\Theta \in \mathbb{C}[[x, \mathbf{y}]]^{n-1}$ has order at least 2 in the $\mathbf{y}$-variables. We may assume $q \geq 0$ since otherwise the vector field $x^{-l} X$ would be non-singular, a case already treated above. Moreover, up to increasing $l$ and decreasing $q$, we may assume also that $A(0) \neq 0$.

Notice that if $q=0$ then $X$ is already in the required form (14) with $l=k$ and $p=0$. We assume that $q \geq 1$. To the vector field $X$ in (16) we can associate the system of $n-1$ formal meromorphic ODEs

$$
x^{q+1} \mathbf{y}^{\prime}=u(x, \mathbf{y})^{-1}(c(x)+A(x) \mathbf{y}+\Theta(x, \mathbf{y})) .
$$

Systems of meromorphic ODEs are extensively studied in the linear case

$$
x^{q+1} \mathbf{y}^{\prime}=B(x) \mathbf{y},
$$

where $q \geq-1$ and $B(x)$ is a matrix with formal coefficients. For such a system, the number $q$ is called the Poincaré rank if $B(0) \neq 0$; in this case, the origin is singular if $q \geq 0$, a regular singularity if $q=0$ and an irregular singularity if $q \geq 1$. We will use the following particular result of this theory (see Turrittin [28] or also Wasow 29] or Balser [4). 
Theorem 6.3 (Turrittin). Consider a formal linear $m$-dimensional system $x^{q+1} \mathbf{y}^{\prime}=$ $B(x) \mathbf{y}$, where $q$ is the Poincaré rank, and assume that $q \geq 1$. Then, after a finite number of transformations of the variables among the following types

- Polynomial linear transformation

$$
L(P(x)): \mathbf{y}=P(x) \widetilde{\mathbf{y}}, P(x) \in \mathcal{M}_{m}(\mathbb{C}[x]) \text { with } P(0) \text { invertible. }
$$

- Shearing transformation

$$
S\left(k_{1}, \ldots, k_{m}\right): \mathbf{y}=\operatorname{diag}\left(x^{k_{1}}, \ldots, x^{k_{m}}\right) \widetilde{\mathbf{y}}, k_{j} \in \mathbb{N}_{\geq 0} .
$$

- Ramification

$$
R(\alpha): \quad x=\widetilde{x}^{\alpha}, \alpha \in \mathbb{N}_{>0} .
$$

the system transforms into a system

$$
x^{p+1} \widetilde{\mathbf{y}}^{\prime}=\left(\mathcal{D}(x)+x^{p} \mathcal{C}+O\left(x^{p+1}\right)\right) \widetilde{\mathbf{y}},
$$

where either $p=0$ and $\mathcal{C} \neq 0$ or $p \geq 1, \mathcal{D}(x)$ is a diagonal matrix of polynomials of degree at most $p-1$ commuting with $\mathcal{C}$ and $\mathcal{D}(0) \neq 0$.

Polynomial linear transformations, shearing transformations and ramifications, as defined in Turrittin's Theorem, will be called T-transformations.

Remark 6.4. Notice that a polynomial linear transformation does not change the Poincaré rank and that a ramification $R(\alpha)$ multiplies it by $\alpha$. The effect of a shearing transformation $S\left(k_{1}, \ldots, k_{m}\right)$ on the Poincaré rank depends on the parameters $k_{1}, \ldots, k_{m}$. Looking carefully at the proof of Theorem 6.3 (see for example 29, section 19), we may observe that the shearing transformations are chosen so that their application never makes the Poincaré rank increase strictly.

We resume the proof of Theorem 6.2. Assume that $X$ is written as in (16). Consider the formal change of variables $\mathbf{y}=\hat{\mathbf{y}}+\bar{\gamma}(x)$, for which $\Gamma=\{\hat{\mathbf{y}}=0\}$, and write $X$ in the variables $(x, \hat{\mathbf{y}})$ as

$$
x^{l}\left[x^{q+1} u(x, \hat{\mathbf{y}}+\bar{\gamma}(x)) \frac{\partial}{\partial x}+(\hat{A}(x) \hat{\mathbf{y}}+\hat{\Theta}(x, \hat{\mathbf{y}})) \frac{\partial}{\partial \hat{\mathbf{y}}}\right]
$$

where $\hat{A}(0) \neq 0$ and $\hat{\Theta}(x, \hat{\mathbf{y}})=O\left(\|\hat{\mathbf{y}}\|^{2}\right)$. The system (17) becomes

$$
x^{q+1} \hat{\mathbf{y}}^{\prime}=u(x, \hat{\mathbf{y}}+\bar{\gamma}(x))^{-1}(\hat{A}(x) \hat{\mathbf{y}}+\hat{\Theta}(x, \hat{\mathbf{y}})) .
$$

Apply Theorem 6.3 to the linear system $x^{q+1} \mathbf{w}^{\prime}=u(x, \bar{\gamma}(x))^{-1} \hat{A}(x) \mathbf{w}$ with $m=$ $n-1$, associated to the vector field

$$
Y=x^{l}\left[x^{q+1} u(x, \bar{\gamma}(x)) \frac{\partial}{\partial x}+\hat{A}(x) \mathbf{w} \frac{\partial}{\partial \mathbf{w}}\right] .
$$

Let us justify that the T-transformations involved are sequences of permissible transformations for $(Y,\{\mathbf{w}=0\})$. This is clear for polynomial linear transformations and for ramifications. On the other hand, a shearing transformation can be viewed as a composition of blow-ups with centers of codimension two. More precisely, $S\left(k_{2}, \ldots, k_{n}\right)$ is obtained by blowing-up $k_{2}$ times the center with equations $\left\{x=w_{2}=0\right\}$ (considering repeatedly the expression $\left.\phi(x, \mathbf{w})=\left(x, x w_{2}, w_{3}, \ldots, w_{n}\right)\right)$, followed by blowing-up $k_{3}$ times the center $\left\{x=w_{3}=0\right\}$, and so on. It suffices to show that such local blow-ups are permissible. Consider one of such centers, for instance $Z=\left\{x=w_{2}=0\right\}$, and let $\phi(x, \mathbf{w})=\left(x, x w_{2}, w_{3}, \ldots, w_{n}\right)$ be the blow-up 
with center $Z$. One can check that the transform of $x^{-l} Y$ by $\phi$, which is a meromorphic vector field, has coefficients with no poles if and only if $x^{-l} Y\left(w_{2}\right)$ belongs to the ideal $I(Z)=\left(x, w_{2}\right)$, i.e. if and only if $Z$ is invariant for $x^{-l} Y$. Observe that if the resulting vector field has poles then the Poincaré rank of the associated linear system will increase and so, by Remark 6.4. we conclude that $Z$ is invariant for $x^{-l} Y$ and thus for $Y$. Moreover, $Y(x)=O\left(x^{l+1}\right)$ and $Y\left(w_{2}\right)=x^{l}\left(\sum_{i=2}^{n} \lambda_{i} w_{i}+O(x)\right)$, where $\lambda_{i} \in \mathbb{C}$ and, being $Z$ invariant for $x^{-l} Y, \lambda_{i}=0$ if $i \neq 2$. We conclude that $\nu_{Z}(Y) \geq l+1=\nu_{0}(Y)$ and hence $Z$ is a permissible center for $(Y,\{\mathbf{w}=0\})$.

Denote by $\Psi:(x, \widetilde{\mathbf{w}}) \mapsto\left(x^{\beta}, \Psi_{2}(x, \widetilde{\mathbf{w}})\right)$ the composition of the T-transformations used in Turrittin's process for $Y$. Then, taking into account the conclusion in Theorem 6.3, $\Psi$ is a reduction of $(Y,\{\mathbf{w}=0\})$ to $\mathrm{RS}$-form. More precisely,

$$
\Psi^{*} Y=x^{k}\left[x^{p+1} \beta^{-1} u\left(x^{\beta}, \bar{\gamma}\left(x^{\beta}\right)\right) \frac{\partial}{\partial x}+\left(\mathcal{D}(x)+x^{p} \mathcal{C}+O\left(x^{p+1}\right)\right) \widetilde{\mathbf{w}} \frac{\partial}{\partial \widetilde{\mathbf{w}}}\right]
$$

with the conditions for $k, p, \mathcal{D}(x)$ and $\mathcal{C}$ stated in Theorem 6.2. Observe that $k+p=\beta(l+q)$.

For any $m$, consider the diffeomorphism $\phi_{m}:\left(\mathbb{C}^{n}, 0\right) \rightarrow\left(\mathbb{C}^{n}, 0\right)$ given by the change of variables $\mathbf{y}=\widetilde{\mathbf{y}}+J_{m} \bar{\gamma}(x)$. The transform $X_{m}$ of $X$ by $\phi_{m}$ is written as

$$
X_{m}=x^{l}\left[x^{q+1} u\left(x, \widetilde{\mathbf{y}}+J_{m} \bar{\gamma}(x)\right) \frac{\partial}{\partial x}+\left(c_{m}(x)+A_{m}(x) \widetilde{\mathbf{y}}+\Theta_{m}(x, \widetilde{\mathbf{y}})\right) \frac{\partial}{\partial \widetilde{\mathbf{y}}}\right],
$$

where $A_{m}(x), \Theta_{m}(x, \widetilde{\mathbf{y}})$ converge to $\hat{A}(x), \hat{\Theta}(x, \widetilde{\mathbf{y}})$ respectively in the Krull topology and $\Theta_{m}(x, \widetilde{\mathbf{y}})=O\left(\|\widetilde{\mathbf{y}}\|^{2}\right)$. Moreover, the transform $\Gamma_{m}$ of $\Gamma$ has order of contact at least $m$ with the $x$-axis $\{\widetilde{\mathbf{y}}=0\}$ and thus the order of $c_{m}(x)$ is at least $m$ (see Remark 3.2). On the other hand, consider for $M \in \mathbb{N}$ the map $\psi_{M}:(x, \mathbf{w}) \mapsto$ $\left(x, \tilde{\mathbf{y}}=x^{M} \mathbf{w}\right)$. Notice that $\psi_{M}$ is a composition of punctual permissible blow-ups for $\left(X_{m}, \Gamma_{m}\right)$ if $m>M+1$. Let $\left(X_{m, M}, \Gamma_{m, M}\right)$ be the transform of $(X, \Gamma)$ by $\psi_{M} \circ \phi_{m}$, when $m>M+1$. Taking into account the effect, mentioned above, of punctual blow-ups in the monomials of positive order in the $\mathbf{y}$-variables, it is not difficult to check that, if $M$ is sufficiently big, then the map $\Psi$ is a sequence of permissible transformations, not only for $(Y,\{\mathbf{w}=0\})$, but also for $\left(X_{m, M}, \Gamma_{m, M}\right)$ (the centers involved have equations of the form $\left\{x=w_{j}=0\right\}$ and the property of being permissible for $\left(X_{m, M}, \Gamma_{m, M}\right)$, by the same arguments used above for $(Y,\{\mathbf{w}=0\})$, only depends on the linear part in each step as long as $x$ divides the non-linear terms).

In order to finish the proof of Theorem 6.2 it suffices to prove that if $m$ and $M$ are sufficiently big then $\Psi$ is a reduction of $\left(X_{m, M}, \Gamma_{m, M}\right)$ to RS-form: the map $\Psi \circ \psi_{M} \circ \phi_{m}$ will then be a reduction of $(X, \Gamma)$ to RS-form. We use Remark 5.2 repeatedly for any of the permissible transformations of the composition $\Psi$ : there exists $N^{\prime}$ such that, given a vector field $W$, if $J_{N^{\prime}}(W)=J_{N^{\prime}}(Y)$ and $\Psi$ is a sequence of permissible transformations for $W$ (with no conditions concerning any formal invariant curve) then

$$
J_{k+p+1}\left(\Psi^{*} W\right)=J_{k+p+1}\left(\Psi^{*} Y\right) .
$$

Now, choose $m, M$ sufficiently big with $m>M+1$ so that $J_{N^{\prime}}\left(X_{m, M}\right)=J_{N^{\prime}}\left(\psi_{M}^{*} Y\right)$. Notice that $\psi_{M}$ is a sequence of permissible blow-ups for $(Y,\{\mathbf{w}=0\})$ and that

$$
\psi_{M}^{*} Y=x^{l}\left[x^{q+1} u(x, \bar{\gamma}(x)) \frac{\partial}{\partial x}+\left(\hat{A}(x)-M x^{q} u(x, \bar{\gamma}(x)) I_{n-1}\right) \mathbf{w} \frac{\partial}{\partial \mathbf{w}}\right] .
$$


Hence, if we put $W_{M}=M x^{l+q} u(x, \bar{\gamma}(x)) I_{n-1} \mathbf{w} \frac{\partial}{\partial \mathbf{w}}$ then $J_{N^{\prime}}\left(X_{m, M}+W_{M}\right)=$ $J_{N^{\prime}}(Y)$ and, by (19) and (18), and since $\Psi$ is also permissible for $X_{m, M}+W_{M}$, we have that $\Psi^{*}\left(X_{m, M}+W_{M}\right)$ has the desired form (14) of Theorem 6.2 with $k+p=\beta(l+q)$. Notice finally, using the expressions (see Theorem 6.3) of the T-transformations involved in the map $\Psi$, that

$$
\Psi^{*}\left(W_{M}\right)=x^{\beta(l+q)} v(x) I_{n-1} \widetilde{\mathbf{w}} \frac{\partial}{\partial \widetilde{\mathbf{w}}},
$$

where $v(0) \neq 0$. We conclude that $\Psi^{*}\left(X_{m, M}\right)$ is also in the form (14) and thus $\Psi$ is a reduction of $\left(X_{m, M}, \Gamma_{m, M}\right)$ to $\mathrm{RS}$-form as wanted (we need to remark that it is possible that $p=0$ and $\mathcal{C}=-v(0) I_{n-1}$ in which case the resulting matrix $\mathcal{C}$ for $\Psi^{*}\left(X_{m, M}\right)$ vanishes; however, in this case, $x^{-(k+1)} \Psi^{*}\left(X_{m, M}\right)$ is non singular and can be treated as we did above). Theorem 6.2 is finished.

\section{Conclusion}

In this final section, we restate and prove Theorem 2 in the introduction.

Given a diffeomorphism $F$ with an invariant formal curve $\Gamma$ not contained in the set of fixed points, we say that $\Gamma$ is well placed for $F$ if there exists a sequence $\Phi$ that reduces $(F, \Gamma)$ to Ramis-Sibuya form as in Theorem 6.1 and such that one of the attracting directions of the transform $(\widetilde{F}, \widetilde{\Gamma})$ is contained in the saddle domain.

Theorem 7.1. Let $F \in \operatorname{Diff}_{1}\left(\mathbb{C}^{n}, 0\right)$ have an invariant formal curve $\Gamma$ not contained in $\operatorname{Fix}(F)$. If $\Gamma$ is well placed for $F$, then there exists at least one attracting parabolic curve for $F$ asymptotic to $\Gamma$.

Proof. By Theorem 3.3 there exists an attracting parabolic curve $\widetilde{\varphi}$ of $\widetilde{F}$ asymptotic to $\widetilde{\Gamma}$. This parabolic curve does not cut the total divisor $E_{\Phi}$ of $\Phi$, since $E_{\Phi}=\operatorname{Fix}(\widetilde{F})$ by Theorem 6.1 Hence, if $\Phi=\phi_{l} \circ \phi_{l-1} \circ \cdots \circ \phi_{1}$ and $\Phi_{j}=\phi_{l} \circ \phi_{l-1} \circ \cdots \circ \phi_{l-j}$ for $j=0, \ldots, l-2$, the map $\varphi_{j}=\Phi_{j} \circ \widetilde{\varphi}$ does not cut either the exceptional divisor $E_{\phi_{l-j-1}}$ of $\phi_{l-j-1}$. Applying recursively Proposition 5.3 for $j=0, \ldots, l-2$, the map $\varphi=\Phi \circ \widetilde{\varphi}$ is a parabolic curve of $F$ asymptotic to $\Gamma$ (notice that we may assume that $\varphi$ is injective restricting the domain of $\widetilde{\varphi}$ by part (ii) of Theorem 3.3).

Notice finally that the remark mentioned in the introduction about finite determinacy of the property of being well placed is a consequence of Remark 5.2

\section{REFERENCES}

[1] Авате, M. The residual index and the dynamics of holomorphic maps tangent to the identity. Duke Math. J. 107 (2001), no. 1, 173-207.

[2] Abate, M; Bracci, F.; Tovena, F. Index theorems for holomorphic self-maps. Ann. of Math. (2) 159 (2004), 819-864.

[3] Abate, M; Tovena, F. Parabolic curves in $\mathbb{C}^{3}$. Abstr. Appl. Anal. 2003, no. 5, 275-294.

[4] BAlSER, W. Formal power series and linear systems of meromorphic ordinary differential equations. Universitext (2000), Springer-Verlag, New York.

[5] Branksma, B. Multisummability of formal power series solutions of nonlinear meromorphic differential equations. Ann. Inst. Fourier (Grenoble) 42 (1992), no. 3, 517-540.

[6] Brochero Martínez, F.E.; Cano, F.; López-Hernanz, L. Parabolic curves for diffeomorphisms in $\mathbb{C}^{2}$. Publ. Mat. 52 (2008), no. 1, 189-194.

[7] Brochero Martínez, F.E.; López-Hernanz, L. Gevrey class of the infinitesimal generator of a diffeomorphism. Astérisque 323 (2009), 33-40.

[8] CAMACHO, C.; SAD, P. Invariant varieties through singularities of holomorphic vector fields. Ann. of Math., 115 (1982), 579-595. 
[9] Cano, J. On the series defined by differential equations, with an extension of the Puiseux polygon construction to these equations. Analysis 13, 103-119 (1993).

[10] Cano, F.; Cerveau, D.; Déserti, J. Théorie élémentaire des feuilletages holomorphes singuliers. Éditions Belin, 2013. Collection "Échelles". ISBN: 978-2-7011-7484-6. 208p.

[11] Cano, F.; Moussu, R.; Rolin, J.-P. Non-oscillating integral curves and valuations. J. Reine Angew. Math., 582 (2005), 107-141.

[12] Cano, F.; Moussu, R.; Sanz, F. Oscillation, spiralement, tourbillonnement. Comm. Math. Helv., 75 (2000), 284-318.

[13] Cano, F.; Moussu, R.; Sanz, F. Pinceaux de courbes intégrales d'un champ de vecteurs analytique. Astérisque, 297 (2004), 1-34.

[14] ÉCAlle, J. Les fonctions résurgentes. Tome III. L'équation du pont et la classification analytique des objects locaux. Publications Mathématiques d'Orsay, 85-5. Université de Paris-Sud, Département de Mathématiques, Orsay, 1985.

[15] Fatou, P. Sur les équations fonctionelles. Bull. Soc. Math. France 47 (1919) 161-271.

[16] Gómez Mont, X.; Luengo, I. Germs of holomorphic vector fields in $\mathbb{C}^{3}$ without a separatrix. Invent. Math. 109, 1992, No. 2, 211-219.

[17] Hakim, M. Analytic transformations of $\left(\mathbf{C}^{p}, 0\right)$ tangent to the identity. Duke Math. J. 92 (1998), no. 2, 403-428.

[18] LeAu, L. Étude sur les équations fonctionelles à une ou à plusieurs variables. Ann. Fac. Sci. Toulouse Sci. Math. Sci. Phys. 11 (1897) 1-110.

[19] López-Hernanz, L. Summable formal invariant curves of diffeomorphisms. Ergodic Theory Dynam. Systems 32 (2012), no. 1, 211-221.

[20] Loray, F. Pseudo-groupe d'une singularité de feuilletage holomorphe en dimension deux. Prépublication IRMAR, 2005.

[21] Malgrange, B.; Ramis, J.P. Fonctions multisommables. Ann. Inst. Fourier, Grenoble, 42 (1992), 353-368.

[22] Panazzolo, D. Resolution of singularities of real-analytic vector fields in dimension three. Acta Math. 197 (2006), no. 2, 167-289.

[23] Ramis, J.P. Les séries $k$-sommables et leurs applications. Complex analysis, microlocal calculus and relativistic quantum theory (Proc. Internat. Colloq., Centre Phys., Les Houches, 1979), pp. 178-199, Lecture Notes in Phys., 126, Springer, Berlin-New York, 1980.

[24] Ramis, J.P.; SibuYA, Y. A new proof of multisummability of formal solutions of non linear meromorphic differential equations. Ann. Inst. Fourier, 33 (1994), 811-848.

[25] Ribón, J. Families of diffeomorphisms without periodic curves. Michigan Math. J., 53, 2 (2005), 243-256.

[26] Rolin, J.-P.; SAnz, F.; Schäfke, R. Quasi-analytic solutions of analytic ODE's and OMinimal Structures. Proc. LMS 95 (2007), 413-442.

[27] Seidenberg, A. Reduction of the singularities of the differential equation $A d y=B d x$. Am. J. of Math., (1968), 248-269.

[28] Turritin, H. L. Convergent solutions of ordinary linear homogeneous differential equations in the neighborhood of an irregular singular point. Acta Math., 93 (1955), 27-66.

[29] Wasow, W. Asymptotic expansions for ordinary differential equations. Intersciencie, New York, 1965 (re-edited Dover Publications Inc. 1987).

Departamento de Matemática, Universidade Federal de Minas Gerais, Belo HoriZONTE, BRAZIL

E-mail address: lorena@mat.ufmg.br

Departamento de Álgebra, Análisis Matemático, Geometría y Topología, UniversiDAD DE VAlladolid, Spain

E-mail address: fsanz@agt.uva.es 